\title{
Efficacy and cultural appropriateness of psychosocial interventions for paediatric burn patients and caregivers: a systematic review
}

\author{
H. M. Williams ${ }^{1,2^{*}}$ (D, K. Hunter ${ }^{3}$, K. Clapham ${ }^{4}$, C. Ryder ${ }^{3,5}$, R. Kimble ${ }^{1,2}$ and B. Griffin 2,6
}

\begin{abstract}
Background: Paediatric burns are highly painful and traumatising injuries that are overrepresented among Aboriginal and Torres Strait Islander people. Paediatric burn patients' pain remains poorly managed by pharmacological interventions, leading to increased anxiety, distress, and trauma in patients and their caregivers. Non-pharmacological psychosocial interventions have been suggested as effective in reducing pain and psychological morbidities among paediatric burn patients and their caregivers; however, their degree of effectiveness and appropriateness for Aboriginal and Torres Strait Islander people is unclear.

Methods: A non-date restricted systematic review was conducted through four databases. Studies published in English assessing psychosocial interventions on paediatric burn patients' physical pain along with theirs and/or their caregiver's anxiety, distress, or trauma symptoms were identified and included in this review. Included studies were assessed for their ability to reduce one of the outcomes of interests and for their reflection of Aboriginal and Torres Strait Islander peoples' perspectives of health.

Results: Of the 3178 identified references, 17 were eligible. These include distraction based techniques $(n=8)$, hypnosis/familiar imagery $(n=2)$, therapeutic approaches $(n=4)$, and patient preparation/procedural control $(n=3)$. Distraction techniques incorporating procedural preparation reduced pain, while discharge preparation and increased 'patient control' reduced patient and caregiver anxiety; and internet based Cognitive Behaviour Therapy reduced short-term but not long-term post-traumatic stress symptoms. No interventions reflected Aboriginal and Torres Strait Islander peoples' perspectives of health; and few targeted caregivers or focused on reducing their symptoms.
\end{abstract}

Conclusions: The development and assessment of psychosocial interventions to appropriately meet the needs of Aboriginal and Torres Strait Islander paediatric burn patients is required.

Keywords: Aboriginal, Torres Strait Islander, Paediatric burns, Psychosocial interventions, Pain, Anxiety, Distress, Psychological trauma

\footnotetext{
* Correspondence: hayley.williams@uq.edu.au

${ }^{1}$ Centre for Children's Burns and Trauma Research, Child Health Research

Centre, The University of Queensland, Graham Street, South Brisbane 4101,

QLD, Australia

${ }^{2}$ Pegg Leditschke Paediatric Burns Centre, Queensland Children's Hospital,

Graham Street, South Brisbane, QLD 4101, Australia

Full list of author information is available at the end of the article
}

(c) The Author(s). 2020 Open Access This article is distributed under the terms of the Creative Commons Attribution 4.0 International License (http://creativecommons.org/licenses/by/4.0/), which permits unrestricted use, distribution, and reproduction in any medium, provided you give appropriate credit to the original author(s) and the source, provide a link to the Creative Commons license, and indicate if changes were made. The Creative Commons Public Domain Dedication waiver (http://creativecommons.org/publicdomain/zero/1.0/) applies to the data made available in this article, unless otherwise stated. 


\section{Background}

Burn injuries cause severe pain [1-5] and can result in psychological trauma [2-4], anxiety $[3,5]$ and distress $[1,3]$. These uniquely challenging injuries affect Aboriginal and Torres Strait Islander people at higher rates than non-Indigenous Australian people. This is highlighted by the Burns Registry of Australia and New Zealand's most recent report that between 2017 and 2018 Aboriginal and Torres Strait Islander people were hospitalised for burn injuries three times more often than non-Indigenous people, and experienced significantly larger burns covering $10-49 \%$ of their Total Body Surface Area (TBSA) [6]. Paediatric specific data indicates similar discrepancies with Aboriginal and Torres Strait Islander children and adolescents experiencing 2.4 times higher rate of hospitalisation from burn injuries than non-Indigenous Australian children between 2011 and 2013 [7].

A unique challenge of these injuries lies within the persistent and debilitating base level of pain that is further intensified by regular procedures undergone for months to years following the initial injury $[8,9]$. The complex nature of burn related pain often results in poor management despite the administration of standard doses of analgesia [8-13] and is particularly difficult to monitor among paediatric burn patients who are less able to articulate the intensity of their own pain [14]. This is further complicated for Aboriginal and Torres Strait Islander people who may not report their pain at all $[15,16]$ or verbally express their pain differently to non-Indigenous Australians [17]. This is particularly concerning as poorly managed pain during hospitalisation strongly predicts burn patients' psychosocial adjustment and overall wellbeing up to two years following treatment and hospital discharge [18].

More specifically, the pain and discomfort experienced by burn patients is associated with increased distress and anxiety [19]. This, in turn, increases the risk of developing other psychological morbidities such as acute stress and post-traumatic stress disorders (PTSD) [19]. The impact of burn related pain and distress is further exacerbated for paediatric burn patients who have a limited understanding of their injury and treatment [20], restricted agency in their care [21], and reduced ability to cope with the unpredictability of a hospital setting [22]. The struggles faced by paediatric burn patients is also greatly felt by their caregivers [20] who often experience overwhelming feelings of guilt, worry, panic, and anxiety whilst struggling with drastic shifts in their parenting role and ability to assist their child [23]. A review of empirical data highlights that $10-20 \%$ of paediatric burn patients and $4-42 \%$ of their caregivers reportedly experience symptoms of PTSD following the burn injury [24]. This highlights the need to effectively treat paediatric burn patients' and their caregivers' anxiety, distress, and psychological trauma. This is further emphasised by the finding that early onset of such psychological morbidities have a high rate of relapsing later in life [25].

The use of non-pharmacological, psychosocial interventions in conjunction with pharmacological analgesia have been suggested for reducing burn related pain and consequential psychological morbidities [26]. Interventions incorporating Gate Control Theory [21] techniques into change of dressing (COD) procedures are suggested as particularly effective in distracting the patient and reducing their ability to concentrate on painful stimuli [27]. Following this theory, virtual reality has shown particularly favourable results on pain management among paediatric burn patients alone [28], and combined with young adult burn patients $[29,30]$. Likewise, music therapy has shown promising effects in reducing anxiety and distress among paediatric burn patients alone [31-33], and combined with adults [34,35]. Other psychosocial interventions utilising cognitive approaches and behavioural strategies have demonstrated similar effects and suitability for use among a wide age range of children and adolescents. More specifically, cognitive approaches including imagery, preparation techniques, information sharing, and coping strategies are suggested as particularly suitable for older children and adolescents [14]. While behavioural strategies including breathing exercises, desensitisation, and positive reinforcement are suggested as particularly suitable for younger children [14].

Several studies have presented the usefulness of such psychosocial interventions, however, no comprehensive comparison or systematic review has been conducted to assess their rigour, effectiveness, or appropriateness in meeting the needs of Aboriginal and Torres Strait Islander paediatric burn patients and their caregivers. This study assessed the effectiveness of any psychosocial intervention in reducing pain and psychological trauma, distress, and/or anxiety among paediatric burn patients and their caregivers generally. Alongside this assessment, we systematically evaluated the appropriateness and applicability of such interventions for use among Aboriginal and Torres Strait Islander families to inform the necessity and directions for future developments of culturally appropriate interventions.

\section{Methods}

\section{Protocol and registration}

Details of the protocol for this systematic review were registered on PROSPERO, the international prospective register of systematic reviews (CRD42018073451) [36].

\section{Eligibility criteria}

The below eligibility criteria were applied (Table 1). Associated search terms were developed in consultation 
with experts from the University of Queensland library (see Additional file 1).

\section{Information sources}

The Cumulative Index to Nursing and Allied Health Literature (CINAHL), Embase, MEDLINE, PubMed, and PsycINFO databases were systematically searched up to November 2019. Database alerts were established at this time and resulting references added as they became available. Reference lists of included manuscripts were hand-screened to identify additional articles not previously captured.

\section{Study selection}

Duplicates were removed prior to the lead author double screening all title and abstract references in the online systematic review software package Covidence, Australia and Argentina (v1086 e0dda871) [37]. An additional reviewer screened $10 \%$ of title and abstract references in Excel to verify accuracy with $91 \%$ agreeance. The lead author screened all full text references and two additional authors screened 50\% each of full text references. Conflicts were resolved via consensus among all authors.

\section{Assessment of cultural components, study quality and risk of bias}

Cultural components were extracted via a form developed in line with Milroy et al.'s the Dance of Life [38], a multidimensional model reflecting the interconnectedness and complex layering of Australian Aboriginal people's perspectives of health and wellbeing. In accordance with the model, data was extracted on five core aspects of health: physical (four items), psychological (three items), social (four items), spiritual (three items), and cultural wellbeing (three items). All items were graded zero (not present) or one (present) with relevant details extracted. The cultural components data form is included as supplementary materials (see Additional file 2).

Risk of bias was assessed against the Cochrane Collaboration's tool for assessing risk of bias [39] (nine items), and an additional three items from the Cochrane suggested risk of bias criteria for EPOC reviews [40] to assess potential intervention contamination, and baseline outcome and characteristic similarities. All risk of bias items were graded zero (high/unclear bias) or one (low risk of bias). Study quality was evaluated by the Downs \& Black Checklist [41] (28 items) to assess reporting, external validity, internal validity, and study power. Power was graded based on the smallest sample group $\left(0=n_{<1}\right.$, $\left.1=\mathrm{n}_{1}-\mathrm{n}_{2}, 2=\mathrm{n}_{3}-\mathrm{n}_{4}, 3=\mathrm{n}_{5}-\mathrm{n}_{6}, 4=\mathrm{n}_{7}-\mathrm{n}_{8}, 5=n_{>8}\right)$, and all other items graded zero (no) or one (yes); possible maximum score of 31. Hooper et al.'s classifications [42] were adapted to provide overall quality rankings of poor ( $\leq 14)$, fair (15-19), good (20-25), or excellent (26-31). Assessment and data extraction were conducted by the lead author for all studies, and independently by two coauthors for $50 \%$ of studies.

\section{Data synthesis}

Meta-analysis was not appropriate due to high heterogeneity among studies. Therefore, data was synthesised narratively, presented in text by outcome (i.e. pain, anxiety, distress, and trauma), and tabulated by intervention type.

\section{Results \\ Study selection}

Database searches returned 3638 abstracts published prior to 18th November 2019. Duplicates were removed and the remaining 1937 abstracts underwent title and abstract screening, resulting in 1821 exclusions and 116 inclusions for full text revision. Exhaustive attempts to obtain full manuscripts was successful for 100 abstracts. Of the remaining 16, five were not able to be acquired and 11 were unavailable in English. Full manuscripts were double screened by the lead author and two coauthors, resulting in a further 82 exclusions. References of the remaining 18 studies were hand-screened, identifying an additional ten abstracts of interest, two of which were included for data extraction (see Additional file 3). Data extraction was not possible for three studies despite thorough attempts to contact study authors to obtain missing data and further information. Data was extracted from the final 17 eligible studies and narratively synthesised. Exclusion rates are outlined in the PRISMA flow diagram (Fig. 1).

Table 1 Eligibility criteria

\begin{tabular}{|c|c|}
\hline Inclusion & Exclusion \\
\hline $\begin{array}{l}\text { 1. Studies focused on unintentional pediatric burn injuries. } \\
\text { 2. Injured children < } 18 \text { years receiving treatment at time of study, and/or their } \\
\text { caregivers. } \\
\text { 3. Assessment of psychosocial interventions }{ }^{a} \\
\text { 4. Randomised control trials (RCT) or non-randomised control trials (NRCT) with } \\
\text { clear comparison groups. } \\
\text { 5. Assessing patient pain and theirs and/or caregiver's anxiety, distress, and/or } \\
\text { trauma symptoms. } \\
\text { 6. Studies published in English with no date restrictions. }\end{array}$ & $\begin{array}{l}\text { 1. Focus on non-burn injuries/illnesses or intentional burn } \\
\text { injuries. } \\
\text { 2. Injured adults }>18 \text { years and/or injured children }<18 \text { years } \\
\text { post burns care. } \\
\text { 3. Assessment of physical interventions i.e. dressings, physical } \\
\text { therapy, massage etc. } \\
\text { 4. Studies with no clear comparison group. } \\
\text { 5. Assessment of any other outcome variable, or studies } \\
\text { measuring only pain. }\end{array}$ \\
\hline
\end{tabular}

${ }^{\mathrm{a}}$ Defined here as any intervention designed primarily to improve psychosocial wellbeing rather than physiological aspects of health 


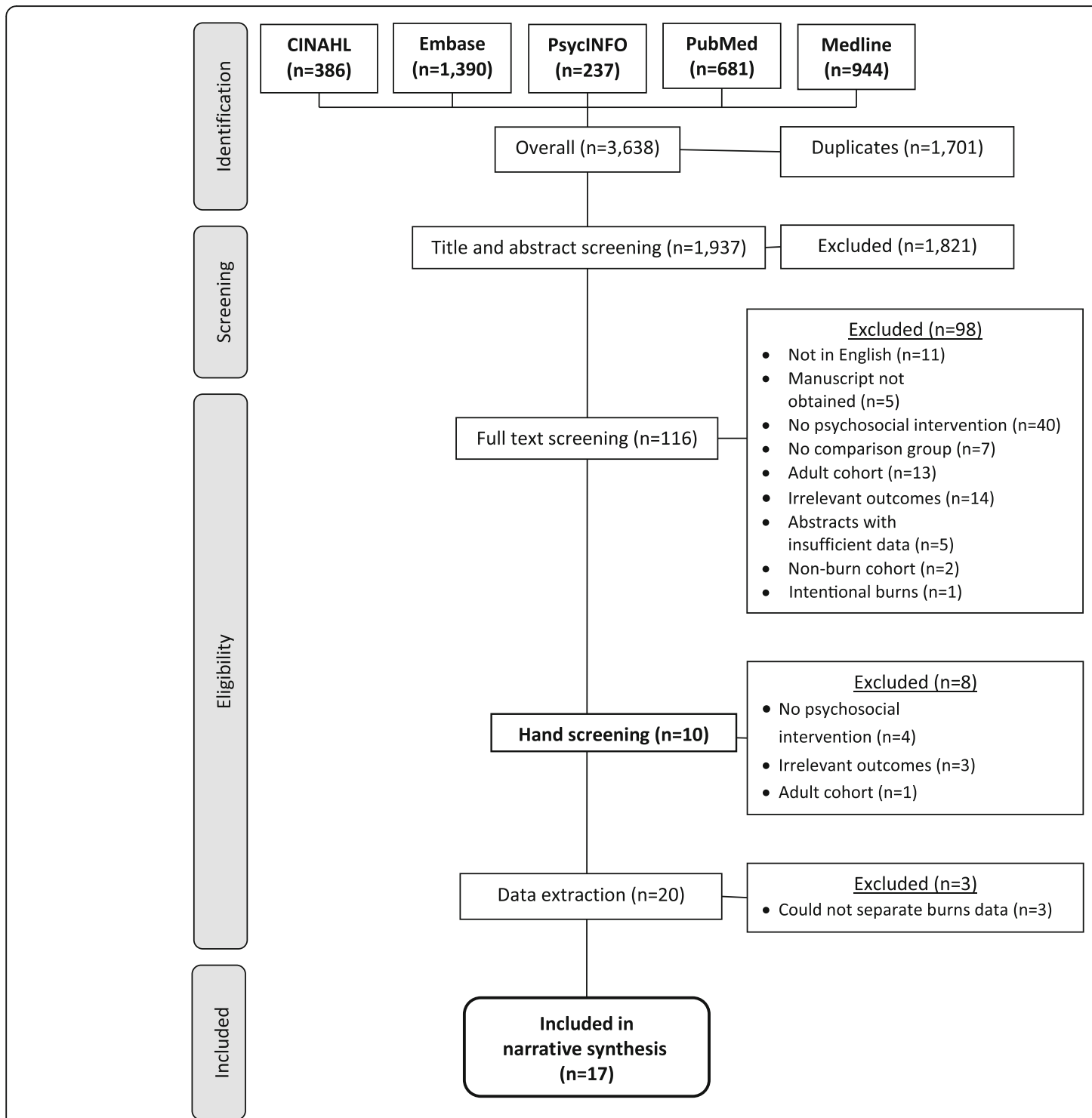

Fig. 1 PRISMA flow chart of inclusion/exclusion rates

\section{Main results}

The participant and study characteristics of included interventions are presented in Tables 2 and 3 respectively. The key findings of included interventions are presented by intervention type in Table 4, and narratively synthesised by outcome below.

\section{Cultural components}

None of the 17 included studies incorporated Australian Aboriginal cultural components as presented in the Dance of Life's model of Australian Aboriginal people's perspectives of health and wellbeing. Blakeney et al.'s social skills psychoeducation was the only study to include Native American children ( $n=2,3 \%)$; however, this study did not assess intervention effect by ethnicity or incorporate any cultural components. Therefore, its ability to meet the needs of First Nations people (respectfully used here in reference to Indigenous peoples globally) could not be ascertained [43]. Moore et al. acknowledged the lack of cultural diversity within their study on medical play; however, this was brief and specific to the inclusion of African American and Hispanic families [55]. Similarly, Chester et al.'s study on hypnotherapy briefly acknowledged the lack of representation of Aboriginal and/or Torres Strait Islander children; however, did not elaborate on any potential implications or outline the ethnic/cultural diversity of included children [46].

Pain

Distraction based interventions had variable effects on patient pain. The Multi-Modal Device (MMD) and $\operatorname{Ditto}^{\mathrm{Tm}}$ devices reduced patient self-reported pain $[44,53$, 
Table 2 Participant characteristics

\begin{tabular}{|c|c|c|c|c|c|c|c|c|c|}
\hline \multirow[t]{2}{*}{ Reference } & \multirow[t]{2}{*}{ Group } & \multirow[t]{2}{*}{ N } & \multirow{2}{*}{$\begin{array}{l}\text { Age } \\
\text { M (SD) }\end{array}$} & \multirow{2}{*}{$\begin{array}{l}\text { Age } \\
\text { Range }\end{array}$} & \multirow{2}{*}{$\begin{array}{l}\text { Male } \\
n(\%)\end{array}$} & \multirow{2}{*}{$\begin{array}{l}\text { TBSA\% } \\
\text { M (SD) }\end{array}$} & \multicolumn{3}{|c|}{ Ethnicity n (\%) } \\
\hline & & & & & & & Reported & Not reported & First Nation \\
\hline \multirow[t]{2}{*}{ Blakeney 2005 [43]. } & I & 32 & $14(1.8)$ & $12-17$ & $9(28)$ & $36.8(25.1)$ & $32(100)$ & $0(0)$ & $2(6)^{*}$ \\
\hline & $C$ & 32 & $14.2(1.9)$ & & $17(53)$ & $44.2(20.6)$ & $32(100)$ & $0(0)$ & $0(0)^{*}$ \\
\hline \multirow[t]{2}{*}{ Brown 2014 [44]. } & । & 47 & $8.3(2.5)$ & $4-13$ & $27(57.5)$ & $1.9(2.2)$ & $47(100)$ & $0(0)$ & $0(0)$ \\
\hline & C & 52 & $8.2(2.7)$ & & $33(63.5)$ & $1.9(2.1)$ & $52(100)$ & $0(0)$ & $0(0)$ \\
\hline \multirow[t]{2}{*}{ Burns-Nader 2017 [45]. } & । & 15 & $7.8(2.3)$ & $4-12$ & $8(53)$ & $9.2(10.3)$ & $15(100)$ & $0(0)$ & $0(0)$ \\
\hline & C & 15 & $7.1(2.8)$ & & $11(73)$ & $6.4(7.5)$ & $15(100)$ & $0(0)$ & $0(0)$ \\
\hline \multirow[t]{2}{*}{ Chester 2018 [46]. } & । & 29 & $8.6(3.4)$ & $4-15$ & $16(59)$ & $1.2(2.0)^{\wedge}$ & $0(0)$ & $29(100)$ & $0(0)^{* *}$ \\
\hline & C & 35 & $7.1(2.7)$ & $4-15$ & $22(63)$ & $1.0(2.0)^{\wedge}$ & $0(0)$ & $35(100)$ & $0(0)^{* *}$ \\
\hline \multirow[t]{2}{*}{ Elliott 1983 [47] } & । & 4 & $8.5(3.5)$ & $5-12$ & $4(100)$ & $21.5(15.0)$ & $3(75)$ & $1(25)$ & $0(0)$ \\
\hline & C & 4 & $6.7(2.1)$ & $5-9$ & $4(100)$ & $32(24.3)$ & $1(25)$ & $3(75)$ & $0(0)$ \\
\hline \multirow[t]{2}{*}{ Foertsch 1998 [48] } & I & 13 & 5.8 & $3-12$ & $12(52)$ & 11.4 & $0(0)$ & $13(100)$ & $0(0)$ \\
\hline & $C$ & 10 & & & & & $0(0)$ & $10(100)$ & $0(0)$ \\
\hline \multirow[t]{2}{*}{ Hyland 2015 [49] } & । & 50 & $2.3(1.5-4.5)^{\wedge}$ & $0-16$ & $25(50)$ & $0.8(0.5-2.0)^{\wedge}$ & $0(0)$ & $50(100)$ & $0(0)$ \\
\hline & C & 50 & $2.2(1.6-3.9)^{\wedge}$ & & $27(54)$ & $0.5(0.5-2.0)^{\wedge}$ & $0(0)$ & $50(100)$ & $0(0)$ \\
\hline \multirow[t]{3}{*}{ Jeffs 2014 [50]. } & $I^{V R}$ & 8 & $14.3(2.0)$ & $10-17$ & $3(38)$ & $7.4(8.5)$ & $6(75)$ & $2(25)$ & $0(0)$ \\
\hline & $1^{P D}$ & 10 & $12.6(2.1)$ & & $8(80)$ & $3.4(3.3)$ & $9(90)$ & $1(10)$ & $0(0)$ \\
\hline & $C$ & 10 & $13.9(2.8)$ & & $8(80)$ & $4.7(6.9)$ & $10(100)$ & $0(0)$ & $0(0)$ \\
\hline \multirow[t]{2}{*}{ Kavanagh 1983 [51] } & I & 4 & $6.3(4.4)$ & $2-11$ & $4(100)$ & $22.7(9.1)$ & $0(0)$ & $4(100)$ & $0(0)$ \\
\hline & C & 5 & $7.1(3.8)$ & $2.5-11.5$ & $3(60)$ & $37.5(27.1)$ & $0(0)$ & $5(100)$ & $0(0)$ \\
\hline \multirow[t]{2}{*}{ Kipping 2012 [52] } & । & 20 & $12.6(1.3)$ & $11-17$ & $13(65)$ & $5.1(6.3)$ & $0(0)$ & $20(100)$ & $0(0)$ \\
\hline & C & 21 & $13.5(1.8)$ & & $15(71)$ & $4.7(4.5)$ & $0(0)$ & $21(100)$ & $0(0)$ \\
\hline \multirow[t]{4}{*}{ Miller 2010 [53] } & $I^{\mathrm{VGD}}$ & 20 & $6.6(2.5)$ & $3-10$ & $12(60)$ & $2.6(1.4)$ & $0(0)$ & $20(100)$ & $0(0)$ \\
\hline & $1^{M M D-D}$ & 20 & $6.6(2.6)$ & & $13(65)$ & $2.8(1.9)$ & $0(0)$ & $20(100)$ & $0(0)$ \\
\hline & IMMD-PP & 20 & $5.5(2.1)$ & & $14(70)$ & $4.3(4.2)$ & $0(0)$ & $20(100)$ & $0(0)$ \\
\hline & C & 20 & $6.1(2.1)$ & & $8(40)$ & $2.5(1.4)$ & $0(0)$ & $20(100)$ & $0(0)$ \\
\hline \multirow[t]{2}{*}{ Miller 2011 [54] } & I & 20 & $6.0(2.0)$ & $3-10$ & $12(60)$ & $2.8(1.0)$ & $20(100)$ & $0(0)$ & $0(0)$ \\
\hline & $C$ & 20 & $5.9(2.5)$ & & $9(45)$ & $2.2(1.1)$ & $20(100)$ & $0(0)$ & $0(0)$ \\
\hline \multirow[t]{4}{*}{ Moore 2015 [55] } & $1^{\text {Patient }}$ & 12 & $3.0^{\wedge}$ & $3-6$ & $6(50)$ & - & $12(100)$ & $0(0)$ & $0(0)$ \\
\hline & $C^{\text {Patient }}$ & 9 & $3.0^{\wedge}$ & $3-5$ & $3(33)$ & - & $9(100)$ & $0(0)$ & $0(0)$ \\
\hline & |Caregiver & 12 & $34^{\wedge}$ & $20-44$ & $1(8)$ & N/A & $0(0)$ & $12(100)$ & $0(0)$ \\
\hline & $C^{\text {Caregiver }}$ & 9 & $28^{\wedge}$ & $23-54$ & $1(11)$ & N/A & $0(0)$ & $9(100)$ & $0(0)$ \\
\hline \multirow[t]{2}{*}{ Quay 1983 [56] } & I & 26 & 5.3 & $0.7-15$ & - & $23(1-81)$ & $0(0)$ & $26(100)$ & $0(0)$ \\
\hline & $C$ & 24 & & & & & $0(0)$ & $24(100)$ & $0(0)$ \\
\hline \multirow[t]{4}{*}{ Sveen 2017 [57] } & Patient & 26 & $5.3(3.5)$ & - & $13(50)$ & $8.5(7.0)$ & $0(0)$ & $26(100$ & $0(0)$ \\
\hline & $C^{\text {Patient }}$ & 23 & $6.4(3.8)$ & & $14(61)$ & $9.9(7.0)$ & $0(0)$ & $23(100)$ & $0(0)$ \\
\hline & |Caregivers & 31 & $36.4(6.6)$ & - & $9(29)$ & N/A & $0(0)$ & $31(100)$ & $0(0)$ \\
\hline & $C^{\text {Caregivers }}$ & 31 & $38.3(5.5)$ & & $11(35)$ & N/A & $0(0)$ & $31(100)$ & $0(0)$ \\
\hline Van der Heijden 2018 [58] & । & 71 & $2.0(13.1-4.1)^{\wedge}$ & $0-13$ & $37(52)$ & $7(4-13) \wedge$ & $0(0)$ & $71(100)$ & $0(0)$ \\
\hline & $C$ & 64 & $1.7(1.3-2.9)^{\wedge}$ & & $32(50)$ & $10(5-15)^{\wedge}$ & $0(0)$ & $64(100)$ & $0(0)$ \\
\hline Whitehead-Pleaux 2006 [59] & । & 8 & - & $6-16$ & $5(36)$ & - & $0(0)$ & $8(100)$ & $0(0)$ \\
\hline & $C$ & 6 & & & & & $0(0)$ & $6(100)$ & $0(0)$ \\
\hline
\end{tabular}

$I$ Intervention, $I^{V R}$ Virtual reality intervention, $I^{P D}$ Passive distraction intervention, $I^{V G D}$ Video game distraction intervention, $I^{M M D-D}$ Multi-modal Device-Distraction intervention, $I^{M M D-P P}$ Multi-modal Device-Procedural Preparation intervention, C Control, M Mean, SD Standard deviation, $\wedge$ Median (IQR), *Native American,

${ }^{* *}$ Aboriginal and Torres Strait Islander/South Sea Islander 
Table 3 Study characteristics

\begin{tabular}{|c|c|c|c|c|c|}
\hline $\begin{array}{l}\text { First } \\
\text { author, } \\
\text { year } \\
\text { [reference] }\end{array}$ & $\begin{array}{l}\text { Study } \\
\text { design, } \\
\text { location }\end{array}$ & Intervention & Control & $\begin{array}{l}\text { Outcome: } \\
\text { measures } \\
\text { (assessor*) }\end{array}$ & Measurement time points \\
\hline $\begin{array}{l}\text { Blakeney } \\
2005 \text { [43]. }\end{array}$ & $\begin{array}{l}\text { RCT, } \\
\text { USA. }\end{array}$ & $\begin{array}{l}n=32^{\wedge 3(9)} \\
\text { 4-day group social skills } \\
\text { workshop based on Changing } \\
\text { Faces REACH OUT, and 'usual' } \\
\text { treatment. }\end{array}$ & $\begin{array}{l}n=32^{\wedge 10(31)} \\
\text { 'Usual' treatment, and follow-up psy- } \\
\text { chological appointments upon re- } \\
\text { quest only. }\end{array}$ & $\begin{array}{l}\text { Anxiety/depression: } \\
C B C L^{(C)}\end{array}$ & $\begin{array}{l}\text { Pre-intervention and } 1 \\
\text { year post-intervention: } \\
C B C L \text {. }\end{array}$ \\
\hline \multirow[t]{2}{*}{$\begin{array}{l}\text { Brown } \\
2014 \text { [44]. }\end{array}$} & \multirow[t]{2}{*}{$\begin{array}{l}\text { RCT, } \\
\text { Australia. }\end{array}$} & \multirow[t]{2}{*}{$\begin{array}{l}n=47^{\wedge 12}(23) \\
\text { Ditto } \\
\text { tion interactive story/game dur- } \\
\text { ing COD. }\end{array}$} & \multirow[t]{2}{*}{$\begin{array}{l}n=52^{\wedge 12(26)} \\
\text { Standard distraction during COD: TV, } \\
\text { videos, books, toys, and caregiver } \\
\text { soothing. }\end{array}$} & \multirow[t]{2}{*}{$\begin{array}{l}\text { Pain: FPS- } R^{(\mathrm{Pt} .)}, H R^{(\mathrm{N})} \text {. } \\
\text { Pain and distress: } \\
\text { FLACC }{ }^{(\mathrm{N})} \text {. } \\
\text { Anxiety: VAS-A } A^{(\mathrm{Pt} .}>8 \\
\text { yrs). } \\
\text { Trauma: } C T S Q^{(\text {Pt. }>6 \text { yrs })}\end{array}$} & $\begin{array}{l}\text { Pre-randomisation: FPS-R, } \\
\text { HR, FLACC, VAS-A. } \\
\text { Pre-removal: FPS-R, FLACC, } \\
\text { VAS-A. } \\
\text { Post-removal: FPS-R, HR, } \\
\text { FLACC, VAS-A, CTSQ }{ }^{(1 \mathrm{st}} \\
\text { (OD) }\end{array}$ \\
\hline & & & & & $\begin{array}{l}\text { Post- application: FPS-R, } \\
H R, \text { FLACC, VAS-A. } \\
\text { During removal and } \\
\text { application: HR. } \\
\text { 3mths post re- } \\
\text { epithelisation: CTSQ. }\end{array}$ \\
\hline $\begin{array}{l}\text { Burns- } \\
\text { Nader } 2017 \\
\text { [45]. }\end{array}$ & $\begin{array}{l}\text { RCT, } \\
\text { USA. }\end{array}$ & $\begin{array}{l}n=15^{\wedge 0}(0) \\
\text { Tablet distraction game, } \\
\text { and CLT support during 2nd } \\
\text { and/or 3rd COD. }\end{array}$ & $\begin{array}{l}n=15^{\wedge 0}(0) \\
\text { Standard distraction, and CLT support } \\
\text { during } 2 \text { nd and/or 3rd COD. }\end{array}$ & $\begin{array}{l}\text { Pain: FACES } S^{(\mathrm{Pt} .)} \text {, nurse's } \\
\text { pain reports }{ }^{(\mathrm{N})}{ }^{(\mathrm{Pt})} \\
\text { Anxiety: } \text { CEMS }^{(\mathrm{Pt})}\end{array}$ & $\begin{array}{l}\text { Prior and during } \\
\text { hydrotherapy: CEMS. } \\
\text { Post-hydrotherapy: FACES, } \\
\text { nurse's pain reports, CEMS. }\end{array}$ \\
\hline $\begin{array}{l}\text { Chester } \\
2018 \text { [46]. }\end{array}$ & $\begin{array}{l}\text { RCT, } \\
\text { Australia. }\end{array}$ & $\begin{array}{l}n=29^{\wedge 0}(0) \\
\text { Hypnosis pre and during COD: } \\
\text { guided imagery, breathing, } \\
\text { muscle relaxation, and }\end{array}$ & $\begin{array}{l}n=35^{\wedge 0}(0) \\
\text { Standard interventions pre and during } \\
\text { COD: parent presence, books, TV, } \\
\text { electronic games, DVDs, toys, bubbles, }\end{array}$ & $\begin{array}{l}\text { Pain: } F P S-R^{(P t)}, F L A C C^{(N)}, \\
N R S^{(C)}, H R^{(R)} . \\
\text { Anxiety: } V A S-A^{(P t .} \geq 8 \text { yrs, } \\
C<8 \text { yrs). }\end{array}$ & $\begin{array}{l}\text { Pre and post-procedure: } \\
\text { FPS-R, FLACC, NRS, VAS-A. } \\
\text { During procedure: FPS-R, } \\
\text { FLACC, NRS. }\end{array}$ \\
\hline & & $\begin{array}{l}\text { permissive and direct hypnotic } \\
\text { suggestions. }\end{array}$ & music, and Ditto ${ }^{\mathrm{TM}}$ PP and distraction. & $\begin{array}{l}\left.\text { Trauma: } \operatorname{CPSS}^{(\text {Pt. }}>7 \mathrm{yrs}\right) \\
Y C P C^{(C<7} \text { yrs) }\end{array}$ & $\begin{array}{l}\text { Pre-medication and post- } \\
\text { application: HR. } \\
\text { 3-months post-injury: } \\
\text { CPSS, YCPC. }\end{array}$ \\
\hline $\begin{array}{l}\text { Elliott } 1983 \\
\text { [47]. }\end{array}$ & $\begin{array}{l}\text { NRCT, } \\
\text { USA. }\end{array}$ & $\begin{array}{l}n=4^{\wedge 0}(0) \\
\text { Stress management during } \\
\text { COD: distraction, breathing, } \\
\text { emotive imagery, and pain } \\
\text { reinterpretation. }\end{array}$ & $\begin{array}{l}n=4^{\wedge 0(0)} \\
S C \text { during COD. }\end{array}$ & $\begin{array}{l}\text { Pain and distress: } \\
\text { BTDS }^{(M S)} \text {. }\end{array}$ & $\begin{array}{l}\text { Removal, first } 15 \text { mins of } \\
\text { hydrotherapy, and during } \\
\text { physical therapy } \\
\text { and dressing re- } \\
\text { application: BTDS. }\end{array}$ \\
\hline $\begin{array}{l}\text { Foertsch } \\
1998 \text { [48]. }\end{array}$ & $\begin{array}{l}\text { RCT, } \\
\text { USA. }\end{array}$ & $\begin{array}{l}n=13^{\wedge \text { Total } 1(43)} \\
\text { Familiar imagery during COD: } \\
\text { focus on childhood memory/ } \\
\text { experience. }\end{array}$ & $\begin{array}{l}n=10^{\wedge \text { Total } 1(43)} \\
\text { Social support during COD: researcher } \\
\text { conversation and encouragement. }\end{array}$ & $\begin{array}{l}\text { Pain and anxiety: } \\
\text { FACES(Pt.'s 3-9 yrs), } \\
\text { VAS } S^{(\text {Pt.'s } 9-12 \text { yrs) }} \\
\text { Distress: } O S B D^{(R)}\end{array}$ & $\begin{array}{l}\text { Baseline and } 15 \text {-s intervals } \\
\text { during procedure: OSBD. } \\
\text { Post-procedure: FACES, } \\
\text { VAS. }\end{array}$ \\
\hline $\begin{array}{l}\text { Hyland } \\
2015 \text { [49]. }\end{array}$ & $\begin{array}{l}\text { RCT, } \\
\text { Australia. }\end{array}$ & $\begin{array}{l}n=50^{\wedge 0}(0) \\
C L T P P \text {, caregiver support and } \\
\text { education, and distraction } \\
\text { during COD. }\end{array}$ & $\begin{array}{l}n=50^{\wedge 0}(0) \\
\text { SC, and minimal distraction during } \\
\text { COD: caregiver, music, and toys/ } \\
\text { electronic devises. }\end{array}$ & 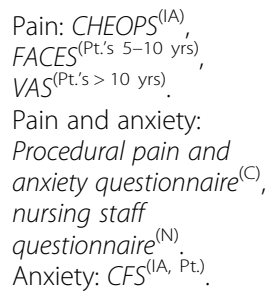 & $\begin{array}{l}\text { Pre-procedure: Procedural } \\
\text { pain and anxiety } \\
\text { questionnaire. } \\
\text { 2-min intervals during } \\
\text { procedure: CHEOPS, CFS. } \\
\text { Post-procedure: FACES, } \\
\text { VAS, Procedural pain and } \\
\text { anxiety questionnaire, CFS. }\end{array}$ \\
\hline $\begin{array}{l}\text { Jeffs } 2014 \\
\text { [50]. }\end{array}$ & $\begin{array}{l}\text { RCT, } \\
\text { USA. }\end{array}$ & $\begin{array}{l}n=8^{\wedge 0}(0) \\
I^{V R}: 3 D \text { interactive program pre } \\
\text { and during COD. }\end{array}$ & $\begin{array}{l}n=10^{\wedge 0}(0) \\
\text { 'Typical' care during COD: standard } \\
\text { nurse communication. }\end{array}$ & $\begin{array}{l}\text { Pain: APPT-WGRS } \\
17 \text { yrs). } \\
\text { Anxiety: STAl-CH } H^{(\text {Pt.) }}\end{array}$ & $\begin{array}{l}\text { Pre-procedure: APPT- } \\
\text { WGRS, STAI-CH. }\end{array}$ \\
\hline
\end{tabular}

$n=10^{\wedge 0}(0)$

$P^{P D}$ : PD (i.e. movie) pre and during COD.

Kavanagh, NRCT, $n=4^{\wedge 0(0)}$

$n=5^{\wedge 0(0)}$

1983 [51]. USA. Max. procedure 'predictability': specific nurse attire, and 'patient control' of 'appropriate' aspects

Min. 'predictability', and medical staff of procedure.

Pain: Nurse reports 0-6 scale ${ }^{(\mathrm{N})}$.

Anxiety: $C B /^{(N, C \text { of pt.'s }}$ $1-3$ yrs)

Pain: $\operatorname{VAS}^{(\text {Pt., C) }}$.
-3 times daily: Nurse reports.

Weekly: CBI. 
Table 3 Study characteristics (Continued)

\begin{tabular}{|c|c|c|c|c|c|}
\hline $\begin{array}{l}\text { First } \\
\text { author, } \\
\text { year } \\
\text { [reference] }\end{array}$ & $\begin{array}{l}\text { Study } \\
\text { design, } \\
\text { location }\end{array}$ & Intervention & Control & $\begin{array}{l}\text { Outcome: } \\
\text { measures }^{\text {(assessor*) }}\end{array}$ & Measurement time points \\
\hline 2012 [52]. & Australia. & $\begin{array}{l}\text { Off-the-shelf VR pre and during } \\
\text { COD. }\end{array}$ & $\begin{array}{l}\text { Standard distraction during COD: TV, } \\
\text { stories, music, and caregiver. }\end{array}$ & $\begin{array}{l}\text { Pain and distress: } \\
\text { FLACC }^{(N)} \text {. }\end{array}$ & $\begin{array}{l}\text { and retrospective post- } \\
\text { removal and application: } \\
\text { VAS, FLACC. }\end{array}$ \\
\hline \multirow[t]{3}{*}{$\begin{array}{l}\text { Miller } 2010 \\
\text { [53]. }\end{array}$} & \multirow[t]{3}{*}{$\begin{array}{l}\mathrm{RCT} \\
\text { Australia. }\end{array}$} & $\begin{array}{l}n=20^{\wedge 2(10)} \\
I^{M M D-P P}: \text { MMD-PP pre-COD, } \\
\text { and standard distraction during } \\
\text { COD. }\end{array}$ & \multirow[t]{3}{*}{$\begin{array}{l}n=20^{\wedge 3}(15) \\
\text { Standard distraction during COD: toys, } \\
\text { TV, and nurse/caregiver interactions. }\end{array}$} & \multirow[t]{3}{*}{$\begin{array}{l}\text { Pain: FACES (P.). } V A S^{(\mathrm{C})} \\
\text { Pain and distress: } \\
\text { FLACC }{ }^{(\mathrm{N})} \text {. }\end{array}$} & \multirow[t]{3}{*}{$\begin{array}{l}\text { Pre and post-removal, pre } \\
\text { and post-application: } \\
\text { FACES, VAS, FLACC. }\end{array}$} \\
\hline & & $\begin{array}{l}n=20^{\wedge} 1^{(5)} \\
I^{M M D-D}: \text { MMD-D interactive } \\
\text { story/game during COD. }\end{array}$ & & & \\
\hline & & 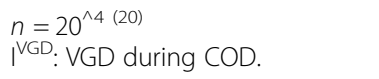 & & & \\
\hline $\begin{array}{l}\text { Miller } 2011 \\
\text { [54]. }\end{array}$ & $\begin{array}{l}\text { RCT, } \\
\text { Australia. }\end{array}$ & $\begin{array}{l}n=20^{\wedge 0}(0) \\
\text { MMD-PP, MMD-D interactive } \\
\text { story/game pre and during } \\
\text { COD. }\end{array}$ & $\begin{array}{l}n=20^{\wedge 0}(0) \\
\text { Standard PP and distraction pre and } \\
\text { during COD. }\end{array}$ & $\begin{array}{l}\text { Pain: FACES } S^{(P \mathrm{t} .)}, V A S^{(\mathrm{C})} \\
\operatorname{HR}^{(\mathrm{N})} \text {. } \\
\text { Pain and distress: } \\
\text { FLACC }{ }^{(\mathrm{N})} \text {. }\end{array}$ & $\begin{array}{l}\text { Pre and post-removal, pre } \\
\text { and post-application: } \\
\text { FACES, VAS, FLACC. } \\
\text { During removal and } \\
\text { application: HR. }\end{array}$ \\
\hline $\begin{array}{l}\text { Moore } \\
2015 \text { [55]. }\end{array}$ & $\begin{array}{l}\text { NRCT, } \\
\text { USA. }\end{array}$ & $\begin{array}{l}n=12^{\wedge 0}(0) \\
C L T \text { MP pre-COD: standard } \\
\text { medical equipment, and } \\
\text { puppets. }\end{array}$ & $\begin{array}{l}n=9^{\wedge 0}(0) \\
\text { SC during COD: standard PP, and } \\
\text { clinical staff verbal explanations. }\end{array}$ & $\begin{array}{l}\text { Pain: } F P S^{(P t .)} \text {. } \\
\text { Pain and distress: } \\
\text { FLACC }{ }^{(R)} \text {. } \\
\text { Anxiety: STAI- } C H^{(C)} \text {. }\end{array}$ & $\begin{array}{l}\text { Pre and post-procedure: } \\
\text { FPS, FLACC, STAI-CH. } \\
\text { Post-removal: FPS. }\end{array}$ \\
\hline $\begin{array}{l}\text { Quay } 1983 \\
\text { [56]. }\end{array}$ & $\begin{array}{l}\text { RCT, } \\
\text { USA. }\end{array}$ & $\begin{array}{l}n=26^{\wedge 0}(0) \\
\text { Discharge preparation weekly } \\
\text { by nurse, written information, } \\
\text { and procedural rehearsal } 3 \text { days } \\
\text { pre-discharge. }\end{array}$ & $\begin{array}{l}n=24^{\wedge 0}(0) \\
\text { Routine instructions } 3 \text { days pre- } \\
\text { discharge. }\end{array}$ & Anxiety: STAI-CH ${ }^{(\mathrm{Pt} ., \mathrm{C})}$. & $\begin{array}{l}\text { 1-day pre-discharge, 1st } \\
\text { follow-up visit: STAI-CH. }\end{array}$ \\
\hline $\begin{array}{l}\text { Sveen } 2017 \\
\text { [57]. }\end{array}$ & $\begin{array}{l}\text { RCT, } \\
\text { Sweden. }\end{array}$ & $\begin{array}{l}n=31^{\wedge 16}(52) \\
\text { Internet based CBT and ACT } \\
\text { support program. }\end{array}$ & $\begin{array}{l}n=31^{\wedge 3(10)} \\
S C \text { during COD. }\end{array}$ & $\begin{array}{l}\text { Post-traumatic stress: } \\
\text { IES-R } R^{(C)}, P S I-S F^{-(C)}, P S S^{(C)}\end{array}$ & $\begin{array}{l}\text { Pre-procedure, post- } \\
\text { procedure, } 3 \text { mths post- } \\
\text { injury, } 12 \text { mths post-injury: } \\
\text { IES-R, PSI-SF, PSS. }\end{array}$ \\
\hline $\begin{array}{l}\text { Van der } \\
\text { Heijden } \\
2018 \text { [58]. }\end{array}$ & $\begin{array}{l}\text { RCT, } \\
\text { South } \\
\text { Africa. }\end{array}$ & $\begin{array}{l}n=71^{\wedge 3}(4) \\
3-5 \text { min MT, and parental } \\
\text { soothing post-COD. }\end{array}$ & $\begin{array}{l}n=64^{\wedge 3(4)} \\
\text { SC during COD, and parental soothing } \\
\text { post-COD. }\end{array}$ & $\begin{array}{l}\text { Pain: COMFORT-B }{ }^{(\mathrm{R})}, \\
\text { FACES }^{(\mathrm{Pt} .)} \text {. } \\
\text { Distress: OSBD-r }{ }^{(\mathrm{R})}, \text { FPS- } \\
R^{(\mathrm{Pt} .)}\end{array}$ & $\begin{array}{l}\text { Pre-procedure, hallway, } \\
\text { entering room: OSBD-r, } \\
\text { COMFORT-B. } \\
\text { Pre and post-procedure: } \\
\text { FPS-R, FACES. }\end{array}$ \\
\hline $\begin{array}{l}\text { Whitehead- } \\
\text { Pleaux } \\
2006 \text { [59]. }\end{array}$ & $\begin{array}{l}\text { RCT, } \\
\text { USA. }\end{array}$ & $\begin{array}{l}n=8^{\wedge 0(0)} \\
\text { MT during COD. }\end{array}$ & $\begin{array}{l}n=6^{\wedge 0(0)} \\
\text { Verbal support and distraction by } \\
\text { music therapists during COD. }\end{array}$ & $\begin{array}{l}\text { Pain: FACES }{ }^{(\mathrm{Pt} .)}, H R^{(\mathrm{R})} \text {. } \\
\text { Behavioral distress: } \\
\text { NAP( }{ }^{(\mathrm{R})} \text {. } \\
\text { Anxiety: Fear } \\
\text { Thermometer }\end{array}$ & $\begin{array}{l}\text { Pre and post-procedure: } \\
\text { FACES, HR, Fear } \\
\text { Thermometer. } \\
\text { During procedure: FACES, } \\
\text { HR, NAPI, Fear } \\
\text { Thermometer. }\end{array}$ \\
\hline
\end{tabular}

*Assessors: Pt patient, $C$ caregiver, $N$ nurse, $R$ researcher, $I A$ independent assessor, $M S$ medical student. $\wedge$ Attrition rate $\mathrm{n}(\%)$. ACT Acceptance and Commitment Therapy, APPT-WGRS Adolescent Paediatric Pain Tool, Word Graphic Rating Scale, BTDS Burn-Treatment Distress Scale, CBCL Children's Behavior Checklist, CBI Children's Behavior Inventory, CBT Cognitive Behavioral Therapy, CEMS Children's Emotional Manifestation Scale, CFS Children's Fear Scale, CHEOPS Children's Hospital of Eastern Ontario Pain Scale, CLT Child Life Therapy, COD Change of dressing, COMFORT-B COMFORT-Behavioral scale, CPSS Child PTSD Symptom Scale, CTSQ Child Trauma Screening Questionnaire, FACES Wong-Baker FACES pain rating scale, FLACC Faces Legs Arms Cry Consolability, FPS Faces Pain Scale, FPS- $R$ Faces Pain Scale-Revised, HR Heart rate, IES-R Impact of Event Scale-Revised, MMD Multi-modal Device, MMD-D Multi-modal Device-Distraction, MMD-PP Multimodal Device-Procedural Preparation, MP Medical play, MT Music therapy, NAPI Nursing Assessment of Pain Index, NRS Numeric Rating Scale, OSBD Observational Scale of Behavioral Distress, OSBD-r Observational Scale of Behavioral Distress-revised, PD Passive distraction, PP Procedural preparation, PSI-SF Parenting Stress Index Short Form, PSS Perceived Stress Scale, SC Standard care, STAI-CH Spielberger State-Trait Anxiety Inventory for Children, VAS Visual Analogue Scale, VAS-A Visual Analog Scale-Anxiety, VGD Video game distraction, VR Virtual reality, YCPC Young Child PTSD Checklist

54], caregiver observations of pain [53, 54], and nurse observations of pain and distress $[53,54]$ when the procedural preparation story "Bobby get's a burn" and interactive distraction games were provided together [44, 54], and separately [53]. The benefits of MMD distraction increased with repeated use and was borderline more effective in reducing nurse observations of pain and distress behaviours when used alone than in combination with procedural preparation [53, 54]. However, less interactive video game distractions were found to reduce self-reported pain and increase caregiver observations of pain over time compared to Multi-Modal Device - 
Table 4 Key results of included studies

\begin{tabular}{|c|c|c|}
\hline Reference & Outcomes & Results \\
\hline \multicolumn{3}{|c|}{ Procedural preparation and distraction } \\
\hline \multirow[t]{4}{*}{ Brown [44] } & Pain & $\begin{array}{l}\text { - FPS-R scores lower in Ditto }{ }^{\mathrm{TM}} \text { than control at post-application of 2nd COD }(\mathrm{MD}=-1.51[\mathrm{Cl}:-2.89,-0.13] p=0.032) \text {. } \\
\text { - HR lowered across } 3 \text { CODs in Ditto }{ }^{\mathrm{TM}} \text { group }(\mathrm{MD}=-4.89[\mathrm{Cl}:-9.69,-0.09], p=0.046) \text {. }\end{array}$ \\
\hline & $\begin{array}{l}\text { Pain and } \\
\text { distress }\end{array}$ & - FLACC scores not reported. \\
\hline & Anxiety & - VAS-A scores lower in Ditto ${ }^{\top M}$ than control at pre-removal (MD $=-1.79$ [Cl:-3.59, -0.01$\left.] p=0.510\right)$ \\
\hline & Trauma & $\begin{array}{l}\text { - Intervention group did not affect CTSQ scores } 1 \text { week post-injury (MD not reported }[\mathrm{Cl}:-1.49,0.87] p=0.602 \text { ) or } \\
3 \text { months post re-epithelialisation (MD not reported }[\mathrm{Cl}:-1.26,2.00] p=0.651 \text { ). }\end{array}$ \\
\hline \multirow[t]{2}{*}{ Burns-Nader [45] } & Pain & $\begin{array}{l}\text { - Intervention group did not affect FACES scores }(p=0.290) \text {. } \\
\text { - Nurse's pain reports lower in tablet group }(M=3.73, S D=0.88) \text { than control }(M=2.93, S D=1.03),(p=0.030) \text {. }\end{array}$ \\
\hline & Anxiety & $\begin{array}{l}\text { - CEMS scores higher in control during }(p=0.001) \text { and after }(p=0.002) \text { hydrotherapy. } \\
\text { - CEMS scores remained higher in control post-procedure }(p<0.050) \text {, tablet group returned to baseline levels } \\
(p=0.570) \text {. }\end{array}$ \\
\hline \multirow[t]{2}{*}{ Miller ${ }^{a}[53]$} & Pain & 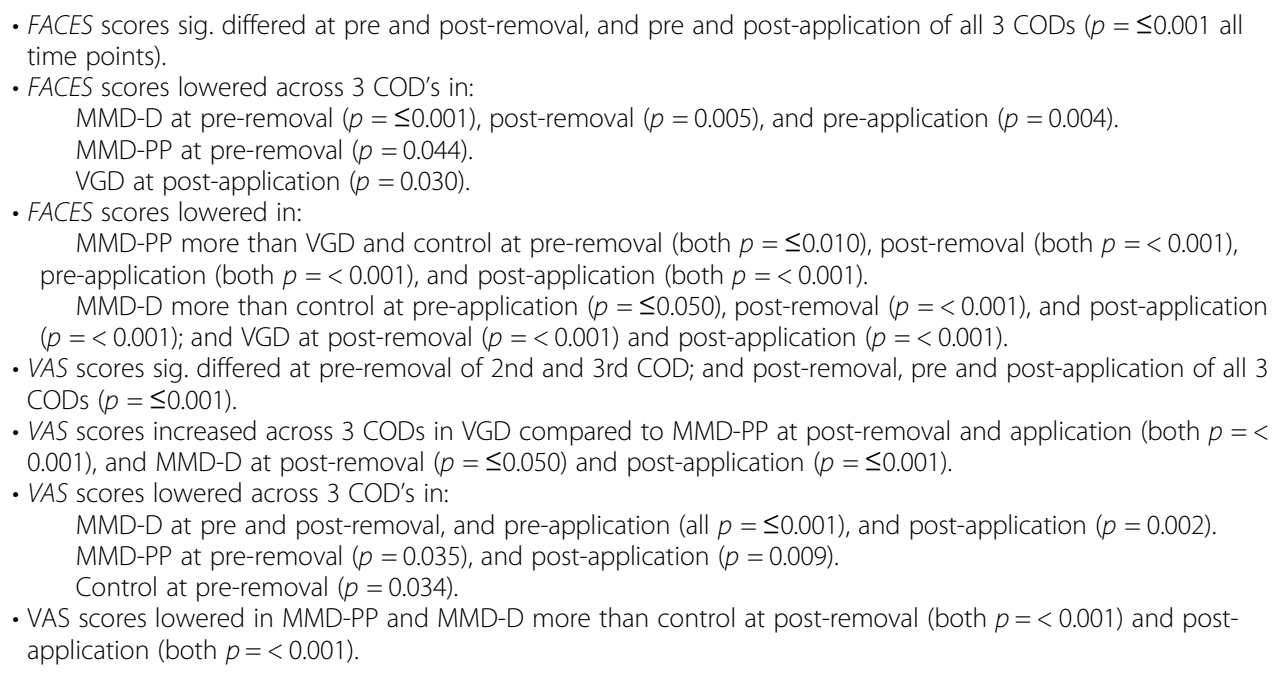 \\
\hline & $\begin{array}{l}\text { Pain and } \\
\text { distress }\end{array}$ & $\begin{array}{l}\text { - FLACC scores sig. differed at pre-removal of } 2 \text { nd and } 3 \text { rd COD }(p=\leq 0.001) \text {; post-removal at } 1 \text { st }(p=0.003) \text {, 2nd, } \\
\text { and 3rd CODs }(p \leq 0.001) \text {; pre-application of 1st }(p=0.010) \text {, } 2 \text { nd, and 3rd CODs }(p \leq 0.001) \text {; and post-application } \\
\text { of all } 3 \text { CODs }(p \leq 0.001) \text {. } \\
\text { - FLACC scores lowered across } 3 \text { COD's in: } \\
\text { MMD-D at post-removal ( } p=0.008) \text {, pre-application }(p=0.047) \text {, and post-application }(p=0.018) \text {. } \\
\text { Control at pre-removal }(p=\leq 0.001) \text {. } \\
\text { - FLACC scores lowered in: } \\
\text { MMD-PP more than control at post-removal }(p=\leq 0.050) \text { and post-application }(p=<0.001) \text {; and VGD at } \\
\text { post-removal }(p=\leq 0.050) \text { and post-application }(p=\leq 0.001) \text {. } \\
\text { MMD-D more than control at post-removal }(p=<0.010) \text {, and post-application }(p=<0.001) \text {; and VGD at } \\
\text { post-removal }(p=\leq 0.010) \text { and post-application }(p=<0.001) \text {. }\end{array}$ \\
\hline \multirow[t]{2}{*}{ Miller ${ }^{\mathrm{b}}$ [54] } & Pain & $\begin{array}{l}\text { - FACES scores lower in MMD than control at pre-removal }(p=0.004) \text {; post-removal, and pre and post-application } \\
\text { (all } p=<0.001), 30 \% \text { reduction. } \\
\text { - VAS scores lower in MMD than control at pre-removal }(p=0.018) \text {, post-removal }(p=0.010) \text {, pre-application ( } p= \\
0.001) \text {, and post-application }(p=<0.001), 30 \% \text { reduction. } \\
\text { - MMD combined sig. lowered pre-removal FACES }(p=0.009) \text { and VAS scores }(p=0.035) \text { compared to MMD-D. } \\
\text { - HR lowered in MMD at removal and application (both } p=0.040 \text { ). }\end{array}$ \\
\hline & $\begin{array}{l}\text { Pain and } \\
\text { distress }\end{array}$ & $\begin{array}{l}\text { - FLACC scores lower in MMD than control at post-removal }(p=<0.001) \text {, pre-application }(p=0.021) \text {, and post-ap- } \\
\text { plication }(p<0.001), 50 \% \text { reduction at removal. } \\
\text { - MMD combined borderline less effective than MMD-D in reducing post-removal FLACC scores }(p=0.050) \text {. }\end{array}$ \\
\hline \multirow[t]{2}{*}{ Jeffs [50] } & Pain & $\begin{array}{l}\text { - APPT-WGRS pre-procedure scores highest in VR, then } \mathrm{SC} \text { and PD }(p=0.041) \text {. } \\
\text { - APPT-WGRS procedure scores lower in VR than PD }(\mathrm{MD}=23.70 \mathrm{~mm}[\mathrm{Cl}: 2.40,45.00] p=0.029) \text {, and } \mathrm{SC}(\mathrm{MD}=9.70 \\
\mathrm{mm}[\mathrm{Cl}:-9.50,28.90] \text { not sig. } p=0.320) \text {. } \\
\text { - Male patients reported less procedural pain }(\mathrm{MD}=32.60 \mathrm{~mm}[\mathrm{Cl}: 14.90,50.20] p=<.001) \text {. }\end{array}$ \\
\hline & Anxiety & - Intervention group did not affect state $(p=0.060)$ or trait anxiety $(p=0.710)$ \\
\hline Kipping [52] & Pain & $\begin{array}{l}\text { - Intervention group did not affect patient VAS scores at dressing removal }(p=0.160) \text { or application }(p=0.400) \text {. } \\
\text { - Intervention group did not affect caregiver VAS scores at dressing removal }(p=0.710) \text { or application }(p=0.750) \text {. }\end{array}$ \\
\hline
\end{tabular}


Table 4 Key results of included studies (Continued)

\begin{tabular}{|c|c|c|}
\hline Reference & Outcomes & Results \\
\hline & $\begin{array}{l}\text { Pain and } \\
\text { distress }\end{array}$ & $\begin{array}{l}\text { - FLACC scores lower in VR }(M=2.90, S D=2.40) \text { than control }(M=4.70, S D=2.50) \text { at dressing removal }(p=0.020) \text {, } \\
\text { but not application }(p=0.230) .\end{array}$ \\
\hline \multirow[t]{2}{*}{$\begin{array}{l}\text { Van der Heijden } \\
\text { [58] }\end{array}$} & Pain & $\begin{array}{l}\text { - Intervention group did not affect COMFORT-B scores before or after intervention }(\mathrm{SMD}=0.04[\mathrm{Cl}:-0.30,0.38] p= \\
\text { 0.990). } \\
\text { - FACES scores lower in MT than SC }(p=0.050) \text {; relevant sample MT }(n=13), \mathrm{SC}(n=5) \text {. }\end{array}$ \\
\hline & Distress & $\begin{array}{l}\text { - Intervention group did not affect OSBD-r scores before or after intervention (SMD }=0.11[C 1:-0.23,0.45] p= \\
0.530) \text {. } \\
\text { - Intervention group did not affect FPS-R scores }(p=0.200) \text {. }\end{array}$ \\
\hline \multirow[t]{3}{*}{$\begin{array}{l}\text { Whitehead- } \\
\text { Pleaux [59] }\end{array}$} & Pain & $\begin{array}{l}\text { - Intervention group did not affect FACES scores before }(p=0.181) \text {, or after procedure }(p=0.345) \text {. } \\
\text { - MD in HR from before to after procedure greatest in control }(p=0.003) \text {. }\end{array}$ \\
\hline & Distress & - NAPI scores higher in MT than control during procedure $(p=0.020)$. \\
\hline & Anxiety & $\begin{array}{l}\text { - Fear Thermometer scores higher in MT than control before }(p=0.043) \text {, and during procedures }(p=0.002) \text {, but } \\
\text { not after }(p=0.228) \text {. }\end{array}$ \\
\hline
\end{tabular}

\section{Hypnosis and guided imagery}

Chester [46] Pain

Pain and distress

Anxiety

Trauma

Foertsch [48] Pain and anxiety

Distress

\section{Therapeutic approaches}

Blakeney [43] Anxiety/ distress

Elliott [47] Pain and distress

Hyland [49] Pain
- Intervention group did not affect overall FPS-R scores before, during, or after any procedure $(p=>0.100)$. - FPS-R scores lower in patients < 8 years at 3rd COD $(\mathrm{MD}=4.71[\mathrm{Cl}: 0.33,9.09] p=0.040)$; relevant sample 3 per group.

- NRS scores lower in hypnotherapy than control at pre-removal of 3rd COD (MD $=-0.91[\mathrm{Cl}:-1.62,-0.20] p=$ 0.010).

- Intervention group did not affect NRS scores at any other time point across 3 CODs $(p=>0.200)$.

- HR lower in hypnotherapy than SC at pre-removal (MD $=-15.20[\mathrm{Cl}:-27.20,-3.20] p=0.010)$ and post-application of $3 r d \operatorname{COD}(\mathrm{MD}=-15.49[\mathrm{Cl}:-28.25,-2.53] p=0.020)$.

- FLACC scores not reported.

- Patients $>8$ years VAS-A scores lower in hypnotherapy than SC at pre-removal of 2nd COD (MD $=-0.80$ [Cl:-1.50, $-0.10] p=0.030$ ).

- Caregiver VAS-A scores for patients < 8 years lower in hypnotherapy than $S C$ at pre-removal of 2 nd $(M D=-1.37$ $[\mathrm{Cl}:-2.57,-0.16] p=0.030)$, and 3rd CODs (MD $=-2.07[\mathrm{Cl}:-3.64,-0.49] p=0.010)$.

- Patient CPSS impairment severity scores lower in hypnotherapy than SC (MD $=0.46[C \mathrm{Cl}:-0.01,0.92] p=0.050)$. - Caregiver YCPC symptom severity scores for children < 7 years higher in hypnotherapy than SC (MD $=0.75[C l$ : $0.05,1.45] p=0.040$ ).

- FACES and VAS scores not analysed due to patient difficulty in comprehending tools.

- Intervention group did not affect OSBD scores between groups $\left(F_{1,9}=0.18, p=>0.500\right)$, or across 4 CODs (exact $\mathrm{F}_{3,18}=1.10, p=<0.300$ ).

- Cry behaviors correlated with verbal resistance at 2 nd $(r[22]=0.77, p=<0.010)$, 3rd $(r[22]=0.56, p=<0.050)$, and 4th CODs ( $r[22]=0.49, p=<0.050)$; with emotional support at 1st ( $[23]=0.58, p=<0.050)$, and 2 nd CODs ( $r$ $[22]=0.88, p=<0.010)$; and with verbal pain at 1st ( $[23]=0.52, p=<0.050)$, and 2 nd $\operatorname{CODs}(r[22]=0.83, p=<$ $0.010)$.

- Female patients displayed higher verbal resistance at baseline (t [21] $=-2.40, p=0.020$ ); and cry behaviors at 2nd-4th COD (t $[20]=-2.26, p=0.030)$.

- $C B C L$ anxious and depressed scores sig. lowered from pre-intervention to 1 year post-intervention in intervention group $(\mathrm{t}=-2.50, p=.017)$ and control $(\mathrm{t}=-2.40, p=.026)$; however not between groups $(p=>0.300)$.

- Group comparisons not possible.

- BTDS scores reduced in intervention group by $25-52 \%$ (mean $=36.7 \%)$ from baseline to post-intervention.

- BTDS scores consistently increased for intervention group in therapist absence.

- Patient's preferred: relaxation, emotive imagery, distraction, imagery of pleasant scenery, and earning tangible reinforcement for coping techniques.

- CLT group received fewer additional analgesic medication during procedure than SC ( $n=6,12 \%$ vs $n=9,18 \%$ ). - Average CHEOPS scores lower in CLT (Mdn = 5.30, IQR: 4.50-6.70) than SC (Mdn =6.00, IQR: 5.40-7.60), (Cl: 0.10, $1.20, p=0.020$ ).

- Nursing staff observed higher pre-procedural pain in CLT than SC (Mdn $=1.00, I Q R: 0.00-2.00$ vs. Mdn $=0.50$, IQR: 0.00-1.00).

- Intervention group did not affect nursing staff observations of procedural pain ( $M d n=2.00$ for both groups).

- FACES scores not reported.

Pain and $\quad$ - CLT caregivers observed higher patient pre-procedural pain than SC caregivers (Mdn =3.50, IQR: 0.00-4.00 vs. anxiety Mdn = 3.00, IQR: 0.00-5.00).

- $C L T$ caregivers observed lower patient procedural pain than SC caregivers (Mdn =2.00, IQR: $0.00-4.00$ vs. Mdn = 
Table 4 Key results of included studies (Continued)

\begin{tabular}{|c|c|c|}
\hline Reference & Outcomes & Results \\
\hline & & $\begin{array}{l}\text { 3.00, IQR: } 1.00-7.00) \text {. } \\
\text { - Intervention group had no affect on caregiver observations of patient pre-procedural anxiety }(\mathrm{Mdn}=2.00 \text {, IQR: } \\
1.00-5.00 \text { vs. Mdn }=2.00 \text {, IQR: } 0.00-5.00 \text { ). } \\
\text { - CLT caregivers observed less patient procedural anxiety than SC caregivers (Mdn }=3.00 \text {, IQR: } 1.00-6.00 \text { vs. } \\
\text { Mdn }=4.30 \text {, IQR: } 1.00-8.00 \text { ). }\end{array}$ \\
\hline & Anxiety & 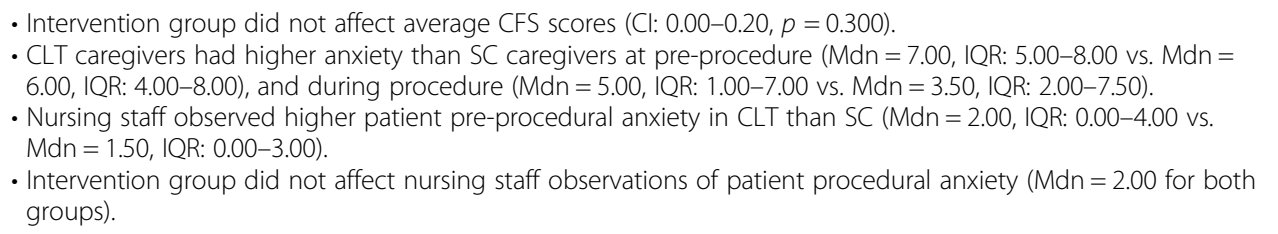 \\
\hline Sveen [57] & $\begin{array}{l}\text { Post-traumatic } \\
\text { stress }\end{array}$ & $\begin{array}{l}\text { - IES- } R \text { scores lower in intervention than control at } 6 \text { weeks post-randomization ( } \beta=-11.50 \text { [SE:3.88] } p=0.003) \\
\text { and } 3 \text { mths post-intervention }(\beta=-7.89[S E: 3.38] p=0.020) \text {. } \\
\text { - Intervention group did not affect IES-R scores at baseline or } 12 \mathrm{mths} \text { post-intervention. } \\
\text { - Intervention group did not affect caregivers PSI-SF or PSS scores at any time point during CODs. } \\
\text { - Caregivers perceived the intervention as informative and meaningful, but time consuming. }\end{array}$ \\
\hline \multicolumn{3}{|c|}{ Preparation \& 'patient control' } \\
\hline \multirow[t]{2}{*}{ Kavanagh [51] } & Pain & $\begin{array}{l}\text { - Intervention group required less analgesic pain medication in } 1 \text { st } 2 \text { weeks of hospitalisation }(p=<0.010) \text {. } \\
\text { - Intervention group received more analgesic medication between CODs }(p=<0.025) \text {. } \\
\text { - Nurse reports not reported. }\end{array}$ \\
\hline & Anxiety & $\begin{array}{l}\text { - Maladaptive symptoms higher in SC than intervention in 1st } 2 \text { weeks of hospitalisation }(p=0.043) \text {. } \\
\text { - Anxiety levels higher in SC than intervention in 1st } 2 \text { weeks, not sig. }(p=0.135) \text {. }\end{array}$ \\
\hline \multirow[t]{3}{*}{ Moore [55] } & Pain & - Intervention group did not affect FPS scores from baseline, during, or post-procedure ( $p=0.717$ ). \\
\hline & $\begin{array}{l}\text { Pain and } \\
\text { distress }\end{array}$ & - FLACC scores lower in MP than SC during CODs (0.50 vs 2.00 respectively), not sig. $(p=0.165)$. \\
\hline & Anxiety & - Intervention group did not affect caregivers state anxiety from baseline to post-procedure ( $p=0.421$ ). \\
\hline Quay [56] & Anxiety & $\begin{array}{l}\text { - Caregivers were able to rehearse treatments and share concerns about returning home. } \\
\text { - STAl-CH scores decreased in intervention caregivers of patients with }>30 \% \text { TBSA burns at discharge }(p=<0.050) \\
\text { and 1st follow-up }(p=<0.050) \text {. } \\
\text { - Intervention group did not affect STAI-CH scores for any patient's, or caregiver of patients with }<30 \% \text { TBSA } \\
\text { burns at discharge or } 1 \text { st follow-up visit. }\end{array}$ \\
\hline
\end{tabular}

APPT-WGRS Adolescent Paediatric Pain Tool, Word Graphic Rating Scale, BTDS Burn-Treatment Distress Scale, CBCL Children's Behavior Checklist, CEMS Children's Emotional Manifestation Scale, CFS Children's Fear Scale, CHEOPS Children's Hospital of Eastern Ontario Pain Scale, Cl 95\% confidence interval, CLT Child Life Therapy, COD Change of dressing, COMFORT-B COMFORT-Behavioral scale, CPSS Child PTSD Symptom Scale, CTSQ Child Trauma Screening Questionnaire, FACES Wong-Baker FACES pain rating scale, FLACC Faces Legs Arms Cry Consolability, FPS Faces Pain Scale, FPS- $R$ Faces Pain Scale-Revised, HR Heart rate, IES- $R$ Impact of Event Scale-Revised, IQR Interquartile range, $M$ Mean, MD Mean difference, Mdn Median, MMD Multi-modal Device, MMD-D Multi-modal Device-Distraction, MMDPP Multi-modal Device-Procedural Preparation, MP Medical play, MT Music therapy, NAPI Nursing Assessment of Pain Index, NRS Numeric Rating Scale, OSBD Observational Scale of Behavioral Distress, OSBD-r Observational Scale of Behavioral Distress-revised, PD Passive distraction, PSI-SF Parenting Stress Index Short Form, PSS Perceived Stress Scale, SC Standard care, SD Standard deviation, SMD Standardised mean difference, STAl-CH Spielberger State-Trait Anxiety Inventory for Children, TBSA Total Body Surface Area, VAS Visual Analogue Scale, VAS-A Visual Analog Scale-Anxiety, VGD Video game distraction, VR Virtual reality, YCPC Young Child PTSD Checklist

Procedure Preparation (MMD-PP), and Multi-Modal Device - Distraction (MMD-D) [53]; while also reducing nurse observations of pain and distress, but not selfreported pain compared to standard distraction [45].

Three-dimensional virtual reality increased self-reported pre-procedural pain and reduced self-reported procedural pain more effectively than passive distractions [50]. While off-the-shelf virtual reality increased nursing staff's observations of pain and distress behaviours, but had no effect on patients' or caregivers' reports of pain [52]. Music therapy reduced self-reported pain compared to standard care when provided immediately following CODs [58, 59]; however, did not affect patients' self-reported pain when provided during CODs $[58,59]$. Likewise, 'medical play' prior to COD commencement did not affect patients' self- reported pain; however, did reduce nursing staff observations of pain and distress behaviour at insignificant levels [55]. In contrast, the use of Child Life Therapy (CLT) reduced patients' pain as observed by caregivers and an independent assessor; and increased nursing staff's observations of pre-procedural pain [49]. Similarly, hypnotherapy reduced pain levels at the third COD as self-reported by patients $<8$ years of age, and caregivers [46]. Stress management during CODs reduced self-reported pain and distress from baseline to post-intervention; however, increased similarly to the control group when the therapist was absent [47]. Further, patients that received increased 'patient control' and 'predictability' required less analgesic medication during the first two weeks of hospitalisations, but more in between CODs [51]. 


\section{Distress}

The MMD and Ditto ${ }^{\mathrm{Tm}}$ devices' procedural preparation and distraction reduced patient self-reported distress $[44,54]$, compared to standard care which increased self-reported distress [44]. Similarly, stress and pain management during CODs reduced patient self-reported distress, but only in the presence of a therapist [47]. In contrast, the use of familiar imagery did not reduce patients' self-reported distress or investigators' observations of distress behaviours [48]. Likewise, music therapy during and following CODs did not reduce nurse observations of distress $[58,59]$, but rather increased observations of distress when performed during COD procedures [59].

\section{Anxiety}

Preparation for dressing procedures or hospital discharge reduced patients' and caregivers' anxiety [51, 55, 56]. Similarly, increased 'predictability' and 'patient control' during CODs reduced patient's anxiety during the first two weeks of hospitalisation; however, not significantly [51]. Hospital discharge preparation reduced anxiety among caregivers of children with burns affecting $\geq 30 \%$ of their TBSA, but did not impact other caregivers' or patients' state anxiety during CODs [56]. Blakeney et al. found that providing psychoeducational programs had similar effects as standard care in reducing patient's self-reported anxiety/depression scores from preintervention to one year post-intervention [43]. Hypnotherapy lowered patients' pre-removal anxiety as reported by patients aged $>8$ years at second COD, and caregivers for patients aged $<8$ years at second and third CODs [46]. Child Life Therapy (CLT) reduced caregivers' observations of patients' procedural anxiety; however increased both caregiver anxiety before and during procedures, and nurse observations of anxiety at preprocedure [49]. The Ditto ${ }^{\mathrm{TM}}$ device lowered self-reported anxiety at pre-removal for patient's $>8$ years [44]. Likewise, tablet based electronic game distraction reduced anxiety during and after COD procedures compared to standard distraction [45]. In contrast, virtual reality did not reduce patient anxiety during CODs [44, 50, 59]; and music therapy provided during CODs increased self-reported anxiety before and during procedure [59].

\section{Trauma}

Few studies measured psychological trauma symptoms and those that did had mixed results [44, 46, 57]. Sveen et al.'s online self-help program reduced patient posttraumatic stress scores six weeks post-baseline and three months post-intervention; however symptoms returned to baseline levels 12 months post-intervention [57]. The online self-help program also did not reduce caregivers' actual or perceived stress at any time [57]. Hypnotherapy significantly lowered patient self-reported trauma impairment severity compared to standard care; however, also increased caregiver's observations of trauma symptoms in their children aged $<7$ years at three months post-injury [46]. The Ditto ${ }^{\mathrm{TM}}$ device did not reduce children's stress or trauma symptoms one week following injury or three months following wound healing [44].

\section{Risk of bias}

Risk of bias assessment is presented in Fig. 2. Allocation blinding was often not possible for participants [45, 47, 50, 53, 54], investigators [43, 45, 47-49, 51, 53, 54, 59], and outcome or data assessors; however, only two studies attempted to reduce such bias with counterrationales [47, 51]. Non-randomised control trials (NRCT) assigned participants based on attendance by month [51], weekday [55], or unspecified time [47]. Selective reporting potentially biased the impression of efficacy in some studies. This was present in Hyland et al. who did not present Wong Baker FACES pain scores [49]; Brown et al. and Chester et al. who did not report nurses' FLACC measures [44, 46]; and Kavanagh who did not present Nurse reports of patient pain [51]. Missing data was acknowledged by Foertsch et al. [48] and Hyland et al. [49] but was not adequately addressed. Elliott \& Olson reported minimal results with no group comparison due to heterogeneity in patient age, time of data collection, and length of hospitalisation [47].

Intervention and control groups differed at baseline. Blakeney et al.'s intervention group contained all $(n=2)$ Native American participants and more females than control [43]. Elliott \& Olson's 'baseline' participants were younger $(\mathrm{M}=6.75 \mathrm{yrs})$ with greater burn TBSA $(\mathrm{M}=$ $32 \%$ ) than intervention (Age $\mathrm{M}=8.50 \mathrm{yrs}$. and TBSA\% $\mathrm{M}=21.50$ ) [47]. Moore et al.'s groups significantly differed in burn location $(p=0.020)$ [55]. Kavanagh's intervention group were all male, younger, with smaller burn TBSA, and shorter hospitalisation than control [51]. Whitehead-Pleaux et al.'s control had significantly lower baseline distress behaviours $(p=0.020)$ and self-reported anxiety $(p=0.040)$ than music therapy; however, the anxiety analysis included an unexplained extra participant [59].

\section{Downs \& Black quality assessment}

Studies were classified as excellent $[46,50,52,54]$, good $[44,45,48,49,53,55,57,58]$, fair $[43,56,59]$, or poor quality $[47,51]$. Three studies had small samples of six or less per group $[47,51,59]$. Some studies did not make clear if participants were recruited from the same population $[43,47]$, or over the same time period [43, 45, 47, $49,59]$; and only four studies adequately described adverse events $[46,50,52,54]$. Two studies did not outline differences in follow-up lengths or perform adequate 


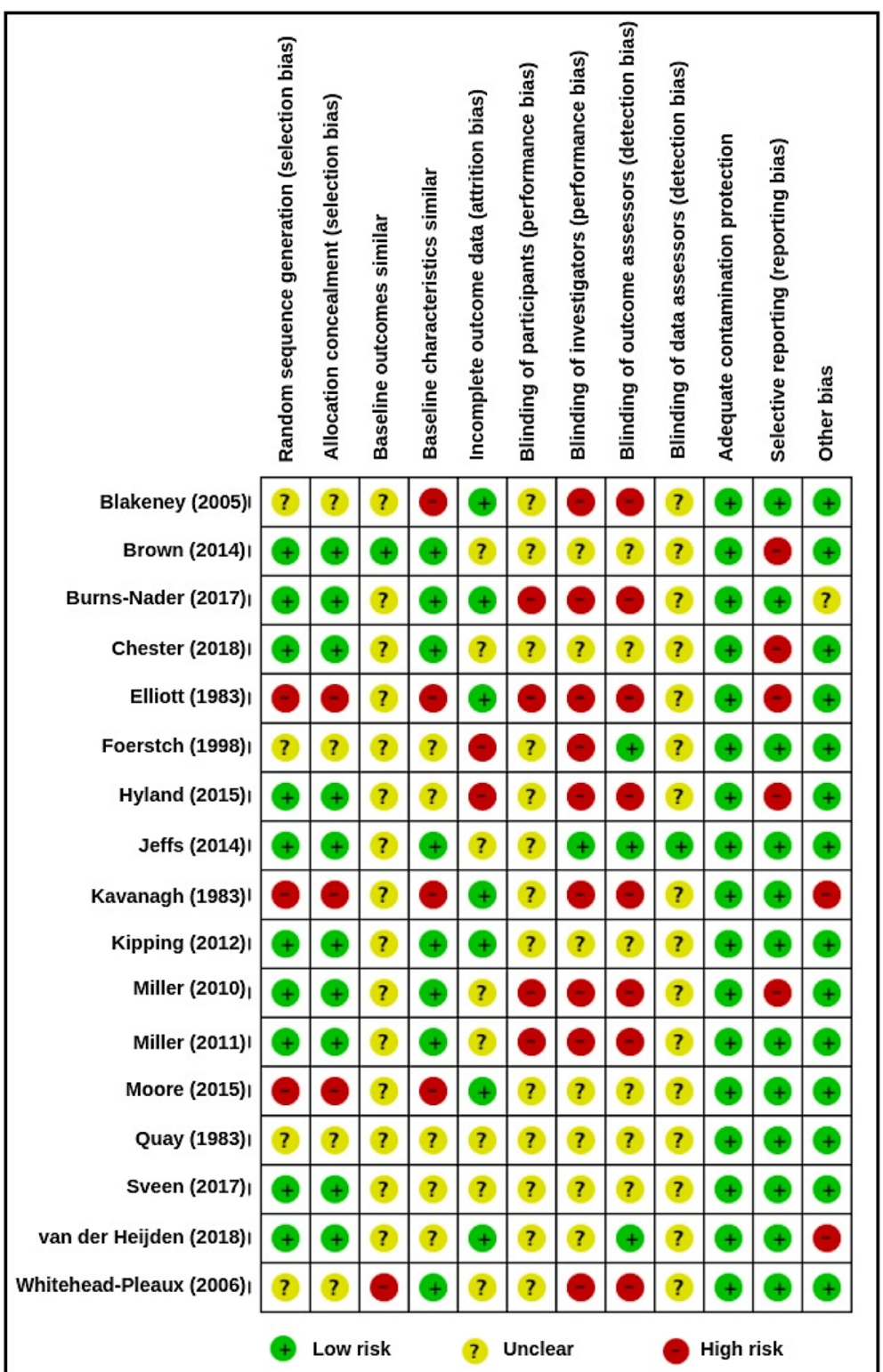

Fig. 2 Risk of bias

adjustments $[47,56]$. Studies did not outline losses to follow-up [50, 51, 59]; or describe the characteristics of the source population [43-59], or those identified as lost to follow-up [43, 44, 47, 48, 53, 56, 57, 59]. Distribution of confounders were not outlined [47, 51,56,60], or clear imbalances inadequately addressed $[43,55]$. Two studies performed seemingly unplanned analysis by burn TBSA [56] and patient age [58]; and data dredging were unclear for two studies [43, 51]. Two studies did not present random variability estimates $[47,51]$, and three did not present actual $p$ values $[47,48,56]$. Furthermore, Kavanagh did not clearly describe outcome measures and stated some participants did not receive both aspects of the intervention [51].

\section{Discussion}

This systematic review highlights a gap in understanding the effectiveness of psychosocial interventions for Aboriginal and Torres Strait Islander paediatric burn patients' and their caregivers. Previous systematic reviews have not accounted for this specific group [60-64], assessed the effects of all psychosocial interventions available [60, 61], or considered psychological trauma as a primary outcome [60-63]. To the authors' knowledge, this systematic review is the first to assess the effectiveness of psychosocial interventions in reducing pain and/or anxiety, distress, and trauma symptoms among paediatric burn patients and their caregivers as well as their relevance to Aboriginal and Torres Strait Islander peoples. 
The appropriateness of the included interventions for Aboriginal and Torres Strait Islander people could not be determined due to the lack of cultural components and First Nations participants. The limited representation of First Nations people is disconcerting given all but one study [57] were conducted in countries with strong First Nations presence; including the United States of America $(n=9,53 \%)$, Australia $(n=6,35 \%)$, and South Africa $(n=1,7 \%)$. The circumstance surrounding the omittance of First Nations people from most studies is unclear; however, some potential reasons may include inaccurate ethnicity records as exemplified by the categorisation of 'lighter skin' vs 'darker skin' by Brown et al. [44], or potential bias in intervention design appeal or accessibility for First Nations people.

Similarly, only two studies provided interventions to caregivers and focused on their symptoms [56, 57]. The limited focus on caregivers is also concerning given the strong evidence of psychological implications of paediatric burn injuries on caregivers [20, 23, 65]. Furthermore, sufficient psychosocial support, education and involvement of caregivers in CODs can build caregivers' competency and aid with their coping during their child's burn treatment $[66,67]$.

The relatively small number of included studies $(n=$ 17) demonstrates the limited scope of formally assessed psychosocial interventions with clear comparison groups. We acknowledge the difficulty of applying rigour and standardisation to psychosocial interventions due to their need for flexibility and adaptability; attributes often key to their responsiveness to individual needs. Standardisation is further compounded by the multi-factorial nature of 'standard' burns care, resulting in varied COD approaches [44], length of time from pain medication to dressing removal [55], days of hospitalisation [56], informal procedural preparation [53], and 'standard' distraction during CODs [46]. However, some studies lacked standardisation of elements outside of 'standard care' that were provided to controls including additional investigator verbal support [48] and active distraction during CODs [51].

The generalisability of included studies was difficult to determine due to poor reporting of source population [43-59], and participants' characteristics [47]. Small sample sizes of $\leq 20$ per group impeded generalisability and power of ten studies [45, 47, 48, 50-55, 59]. Further, some studies excluded families involved with child protection services or prior Suspected Child Abuse and Neglect reports [44, 46, 52]; patients diagnosed with an impairment or Autism Spectrum Disorder [44]; and patients receiving initial CODs in theatre [46], requiring skin grafts, or other diagnosed medical conditions [44]. Other studies restricted inclusion by verbal communicability, inadvertently limiting representation of younger children [59] and non-English speaking families [44, 52]. And other studies demonstrated bias towards families with higher education $[55,57]$ and 'socio-economic status' [44], or with married/cohabitating caregivers and low family conflicts or symptoms of PTSD [57]. In contrast, Kavanagh's intervention participants all reported a history of 'family disorganisation' or psychopathological symptoms, confounding factors to the intervention success [51]. And of particular concern, two included interventions were not tested among females due to gender imbalances between groups or lack of female participation $[47,51]$. Potential gender differences in intervention experience or primary outcomes was not considered by any of the included studies.

This review was limited to studies available in English and with randomised control trial (RCT) and NRCT designs, resulting in the exclusion of seven otherwise relevant studies. These excluded studies were generally reflective of this review's findings; however, three reported on interventions not captured here [68-70]. One study found art therapy effective in allowing paediatric burn patient's to express their traumas [70], another found the combination of art and play therapy reduced patient anxiety [68], and the other found group therapy reduced caregiver anxiety [69]. It is highly recommended that the results of these excluded studies be considered when developing future interventions.

Authors of the included studies often criticised quantitative measures for being relatively subjective $[49,58]$ and heavily reliant on self-reports and structured assessments [58]. Studies reported the Fear Thermometer [59] and Wong-Baker FACES scale [48] to be confusing and difficult for young children to understand, despite being validated for use among children. Likewise, FLACC behavioural pain scale was heavily criticised as inappropriate for children aged 9+ years whom are less likely to display observable signs of distress and pain [48], and for its inability to capture pain and distress differences between groups [55]. Similarly, the Achenbach Child Behavioral Checklist [43], State-Trait Anxiety Inventory [56], and anxiety tool used by Kavanagh [51] were reportedly too general to accurately capture paediatric burn patients' anxiety during CODs.

Despite these challenges, the included interventions demonstrated that procedural distraction via hand-held devices are effective in reducing patient's pain [44, 45, 53, 54]; however, less effective when only games were available [45] opposed to procedural preparation stories as offered by the Ditto ${ }^{\mathrm{TM}}[44]$ and MMD devices $[53,54]$. This indicates that procedural information provided at any time during procedures can reduce patient pain. This is supported by Damanhuri et al.'s finding that active distraction incorporating additional procedural information and encouragement was far more effective 
than passive distraction such as music therapy or games [14]. However, this review also found that distraction techniques did not reduce paediatric patient's anxiety $[44,50,59]$, or trauma symptoms [44] regardless of incorporation of procedural preparation. In contrast, therapeutic approaches were effective in reducing psychological morbidities among patients and caregivers. In particular, CLT reduced caregiver and independent assessor's observations of patient pain, and caregiver's observations of patient anxiety [49]. An online self-help program was the only intervention found to effectively reduce patient trauma symptoms [57]. Similarly, incorporating stress and pain management into CODs reduced patient distress but was not sustained in the therapist's absence [47]. The results of the included studies should be interpreted with consideration of potential bias due to difficulties in intervention allocation blinding [43-59].

\section{Conclusion}

This review returned a limited number of interventions that effectively reduced paediatric burn patient and caregiver psychological morbidities. The scarcity of work on reducing psychological trauma symptoms is particularly disconcerting given the volume of work emphasising the highly traumatising nature of burn injuries for both patients and families [2-4]. This highlights a need for additional work to better support and prepare caregivers for their vital role in providing security and comfort to their children during procedures. Of main concern to this review, the well-documented overrepresentation of Aboriginal and Torres Strait Islander paediatric burn patients was not reflected in the included studies nor were their perspectives on health and wellbeing. This lack of representation highlights the urgency for psychosocial interventions to be developed in partnership with and assessed among Aboriginal and Torres Strait Islander families. Finally, it is suggested that the effects of the included psychosocial interventions be further explored within broader healthcare settings and contexts; in particular, distraction featuring procedural information, CLT, stress and pain management, discharge preparation, and online self-help programs.

\section{Supplementary information}

Supplementary information accompanies this paper at https://doi.org/10. 1186/s12889-020-8366-9.

Additional file 1. Study search terms. Table outlining the search terms developed by the lead author in consultation with experts from the University of Queensland library.

Additional file 2. Data extraction points. Table outlining the topic areas and data points extracted from all applicable studies during the full text review stage.

Additional file 3. Data extraction form adapted from the Dance of Life. Table outlining the criteria for data point extraction developed in accordance with the Dance of Life and utilised on all applicable studies during the full text review.

\section{Abbreviations}

ACT: Acceptance and Commitment Therapy; APPT-WGRS: Adolescent Paediatric Pain Tool, Word Graphic Rating Scale; BTDS: Burn-Treatment Distress Scale; C: Control; CBCL: Children's Behavior Checklist; CBI: Children's Behavior Inventory; CBT: Cognitive Behavioral Therapy; CEMS: Children's Emotional Manifestation Scale; CFS: Children's Fear Scale; CHEOPS: Children's Hospital of Eastern Ontario Pain Scale; Cl: 95\% confidence interval; CINAHL: Cumulative Index to Nursing and Allied Health Literature; CLT: Child Life Therapy; COD: Change of dressing; COMFORT-B: COMFORT-Behavioral scale; CPSS: Child PTSD Symptom Scale; CTSQ: Child Trauma Screening Questionnaire; FACES: Wong-Baker FACES pain rating scale; FLACC: Faces Legs Arms Cry Consolability; FPS: Faces Pain Scale; FPS-R: Faces Pain ScaleRevised; HR: Heart rate; I: Intervention; IES-R: Impact of Event Scale-Revised; $I^{\text {MMD-D: }}$ Multi-modal Device-Distraction intervention; $I^{\text {MMD-PP. }}$ : Multi-modal Device-Procedural Preparation intervention; ${ }^{P D}$ : Passive distraction intervention; IQR: Interquartile range; I $^{\mathrm{VGD}}$ : Video game distraction intervention; IVR: Virtual reality intervention; M: Mean; MD: Mean difference; Mdn: Median; MMD: Multi-modal Device; MMD-D: Multi-modal DeviceDistraction; MMD-PP: Multi-modal Device-Procedural Preparation; MP: Medical play; MT: Music therapy; NAPI: Nursing Assessment of Pain Index; NRCT: Non-randomised Control Trial; NRS: Numeric Rating Scale; OSBD: Observational Scale of Behavioral Distress; OSBD-r: Observational Scale of Behavioral Distress-revised; PD: Passive distraction; PP: Procedural preparation; PSI-SF: Parenting Stress Index Short Form; PSS: Perceived Stress Scale; PTSD: Post-traumatic Stress Disorder; RCT: Randomised Control Trial; SC: Standard care; SD: Standard deviation; SMD: Standardised mean difference; STAI-CH: Spielberger State-Trait Anxiety Inventory for Children; TBSA: Total Body Surface Area; VAS: Visual Analogue Scale; VAS-A: Visual Analog Scale-Anxiety; VGD: Video game distraction; VR: Virtual reality; YCPC: Young Child PTSD Checklist

\section{Acknowledgements}

The contributions of Christine Dalais from the Learning and Research Services at the University of Queensland's Learning and Research Services is gratefully acknowledged for her guidance and contributions to the reviews search strategy.

\section{Authors' contributions}

$\mathrm{HW}, \mathrm{BG}, \mathrm{KH}$, and $\mathrm{KC}$ conceptualised the review. HW conducted all search strategies and hand screening of references. HW double screened all references for title and abstract; CR screened 10\% of references for title and abstract. HW screened and extracted data for all full text references; BG and $\mathrm{KH}$ screened and extracted data for $50 \%$ each of full text references. HW performed the narrative data synthesis. HW led and BG, KH, KC, and RK contributed to the data interpretation. All authors contributed to the drafting and revision of the manuscript, approved the final version, and agree to be accountable for all aspects of the work. All authors read and approved the final manuscript.

\section{Funding}

Hayley Williams is supported by Postgraduate Scholarships from the National Health and Medical Research Council and Children's Hospital Foundation. Kate Hunter is supported by a NSW Health Early-Mid Career Research Fellowship. Courtney Ryder is supported by a Postgraduate Scholarship from the National Health and Medical Research Council. The funding bodies had no role in the design of this review, the collection, analysis, or interpretation of data, or in writing the manuscript.

Availability of data and materials

Not applicable.

Ethics approval and consent to participate Not applicable.

Consent for publication Not applicable. 


\section{Competing interests}

The authors declare that they have no competing interests.

\begin{abstract}
Author details
${ }^{1}$ Centre for Children's Burns and Trauma Research, Child Health Research Centre, The University of Queensland, Graham Street, South Brisbane 4101, QLD, Australia. ${ }^{2}$ Pegg Leditschke Paediatric Burns Centre, Queensland Children's Hospital, Graham Street, South Brisbane, QLD 4101, Australia. ${ }^{3}$ Injury Division, The George Institute for Global Health, University of New South Wales, King Street, Sydney 2042, NSW, Australia. ${ }^{4}$ Ngarruwan Ngadju First Peoples Health and Wellbeing Research Centre, Australian Health Services Research Institute, The University of Wollongong, Wollongong 2522, NSW, Australia. ${ }^{5}$ College of Medicine \& Public Health, Southgate Institute for Health Society and Equity, Flinders University, Registry Road, Bedford Park 5042, SA, Australia. 'School of Nursing, Queensland University of Technology, Ring Road, Brisbane 4059, QLD, Australia.
\end{abstract}

Received: 5 December 2019 Accepted: 17 February 2020

Published online: 04 March 2020

\section{References}

1. Gandhi M, Thomson C, Lord D, Enoch S. Management of pain in children with burns. Int J Pediatr. 2010;2010:1-9. https://doi.org/10.1155/2010/825657.

2. Gürol A, Polat S, Akçay M. Itching, pain, and anxiety levels are reduced with massage therapy in burned adolescents. J Burn Care Res. 2010;31(3):429-32. https://doi.org/10.1097/BCR.0b013e3181db522c.

3. Maron M, Bush J. Burn injury and treatment pain. In: Bush JP, Hawkins SW, editors. Child pain. New York: Springer; 1991. p. 275-95.

4. Stoddard F, Sheridan R, Saxe G, King B, King B, Chedekel D, et al. Treatment of pain in acutely burned children. J Burn Care Rehabil. 2002;23(2):135-56.

5. Weinberg K, Birdsall C, Vail D, Marano M, Petrone S, Hani Mansour E. Pain and anxiety with burn dressing changes: patient self-report. J Burn Care Rehabil. 2000:21(2):157-61.

6. Tracy L, McInnes J, Gong J, Karunakaran R, Gabbe B. Burns registry of Australia and New Zealand 9th annual report. Melbourne: Monash University; 2019

7. Pointer S. Hospitalised injury in Aboriginal and Torres Strait Islander children and young people 2011-13. Canberra: AlHW; 2016. Contract No.: Cat. no. INJCAT 172

8. Abdi S, Zhou Y. Management of pain after burn injury. Curr Opin Anaesthesiol. 2002;15:563-7.

9. Perry $\mathrm{S}$, Heidrich $\mathrm{G}$, Ramos E. Assessment of pain by burn patients. J Burn Care Rehabil. 1981;2(6):322-6. https://doi.org/10.1097/00004630-19811100000004.

10. Choinière $M$, Melzack R, Rondeau J, Girard N, Paquin M. The pain of burns: characteristics and correlates. J Trauma. 1989:29(11):1531-9.

11. Heidrich G, Perry S, Amand R. Nursing staff attitudes about burn pain. J Burn Care Rehabil. 1981;2(5):259-61. https://doi.org/10.1097/00004630-19810900000005.

12. Melzack R. The tragedy of needless pain. Sci Am. 1990;262(2):27-33.

13. Summer G, Puntillo K, Miaskowski C, Green P, Levine J. Burn injury pain: the continuing challenge. J Pain. 2007;8(7):533-48. https://doi.org/10.1016/j. jpain.2007.02.426.

14. Damanhuri N, Enoch S. Assessment and non-pharmacological management of pain in children with burns. In: Mostofsky D, editor. The handbook of behavioral medicine. Hoboken: Wiley; 2014.

15. McGrath P. 'The biggest worry...' research findings on pain management for Aboriginal peoples in Northern Territory, Australia. Rural Remote Health. 2006;6(549):1-14.

16. Strong J, Nielsen M, Williams M, Huggins J, Sussex R. Quiet about pain: experiences of aboriginal people in two rural communities. Aus J Rural Health. 2015;23:181-4. https://doi.org/10.1111/ajr.12185.

17. Fenwick C. Assessing pain across the cultural gap: central Australian indigenous peoples' pain assessment. Contemp Nurse. 2006;22(2):218-27. https://doi.org/10.5172/conu.2006.22.2.218.

18. Patterson D, Tininenko J, Ptacek J. Pain during burn hospitalization predicts long-term outcome. J Burn Care Res. 2006;27(5):719-26. https://doi.org/10. 1097/01.BCR.0000238080.77388.FE.

19. De Young A, Kenardy J, Cobham V, Kimble R. Prevalence, comorbidity and course of trauma reactions in young burn-injured children. J Child Psychol Psychiatry. 2012;53(1):56-63.
20. Rivlin E. The psychological trauma and management of severe burns in children and adolescents. Br J Hosp Med. 1988:40(3):210-5.

21. Schneider F. Assessment and evaluation of audio-analgesic effects on the pain experience of acutely burned children during dressing changes. Ohio: University of Cincinnati; 1982

22. Tarnowski K. Behavioral aspects of pediatric burns. New York: Plenum Press; 1994

23. Rivlin E, Faragher E. The psychological sequelae on mothers of thermally injured children and adolescents: future directions: part 3. Dev Neurorehabil. 2007;10(2):183-90

24. Bakker A, Maertens K, Van Son M, Van Loey N. Psychological consequences of pediatric burns from a child and family perspective: a review of the empirical literature. Clin Psychol Rev. 2013;33:361-71.

25. Hammen C, Brennan P, Keenan-Miller D, Herr N. Early onset recurrent subtype of adolescent depression: clinical and psychosocial correlates. J Child Psychol Psychiatry. 2008;49(4):433-40. https://doi.org/10.1111/j.14697610.2007.01850x

26. Connor-Ballard P. Understanding and managing burn pain: part 2. Am J Nurs. 2009;109(5):54-63.

27. McCaul K, Malott J. Distraction and coping with pain. Psychol Bull. 1984; 95(3):516-33.

28. Chan E, Chung J, Wong T, Lien A, Yung Yang J. Application of a virtual reality prototype for pain relief of pediatric burn in Taiwan. J Clin Nurs. 2007;16:786-93.

29. Faber A, Patterson D, Bremer M. Repeated use of immersive virtual reality therapy to control pain during wound dressing changes in pediatric and adult burn patients. J Burn Care Res. 2013;34(5):563-8. https://doi.org/10. 1097/BCR.0b013e3182777904.

30. Hoffman H, Patterson D, Carrougher G, Sharar S. Effectiveness of virtual reality-based pain control with multiple treatments. Clin J Pain. 2001;17: 229-35

31. Edwards J. "You are singing beautifully": music therapy and the debridement bath. Arts Psychother. 1995;22(1):53-5.

32. Prensner J, Yowler C, Smith L, Steele A, Fratianne R. Music therapy for assistance with pain and anxiety management in burn treatment. J Burn Care Rehabil. 2001:22(1):83-8.

33. Robb S. Techniques in song writing: restoring emotional and phsyical well being in adolescents who have been traumatically injured. Music Ther Perspect. 1996;14:30-7. https://doi.org/10.1093/mtp/14.1.30.

34. Fratianne R, Prensner J, Huston M, Super D, Yowler C, Standley J. The effect of music-based imagery and musical alternate engagement on the burn debridement process. J Burn Care Rehabil. 2001;22(1):47-53.

35. Tan X, Yowler C, Super D, Fratianne R. The efficacy of music therapy protocols for decreasing pain, anxiety, and muscle tension levels during burn dressing changes: a prospective randomized crossover trial. J Burn Care Res. 2010;31(4):590-7. https://doi.org/10.1097/BCR.0b013e3181e4d71b.

36. Williams H, Hunter K, Ryder C, Clapham K, Kenardy J, Kimble R, et al. A systematic review of psychosocial interventions for paediatric burn patients and caregivers effects on pain or psychological morbidities and appropriateness for Aboriginal and Torres Strait Islander Australians., http://www.crd.york.ac.uk/PROSPERO/ display record.php?ID=CRD42018073451; 2018].

37. Covidence. Covidence 2019 https://www.covidence.org/home. [accessed 8 Jan 2019].

38. Milroy $H$. The dance of life. Unpublished conference proceedings presented at AIDA symposium 2002; Sydney, NSW.

39. The Cochrane Collaboration. Table 8.5.d: criteria for judging risk of bias in the 'risk of bias' assessment tool. In: Higgins J, Green S, editors. Cochrane handbook for systematic reviews of interventions; 2011.

40. Cochrane Effective Practice and Organisation of Care (EPOC). Suggested risk of bias criteria for EPOC reviews.: EPOC Resources for review authors; 2017.

41. Downs S, Black N. The feasibility of creating a checklist for the assessment of the methodological quality both of randomised and non-randomised studies of health care interventions. J Epidemiol Community Health. 1998; 52:377-84.

42. Hooper P, Jutai JW, Strong G, Russell-Minda E. Age-related macular degeneration and low-vision rehabilitation: a systematic review. Can J Ophthalmol. 2008;43(2):180-7. https://doi.org/10.3129/i08-001.

43. Blakeney P, Thomas C, Holzer C, Rose M, Berniger F, Meyer W. Efficacy of a short-term, intensive social skills training program for burned adolescents. J Burn Care Rehabil. 2005;26(6):546-56. https://doi.org/10.1097/01.bcr. 0000185455.81677.a2. 
44. Brown N, Kimble R, Rodger S, Ware R, Cuttle L. Play and heal: randomized controlled trial of ditto intervention efficacy on improving reepithelialization in pediatric burns. Burns. 2014;40(2):204-13. https://doi.org/ 10.1016/j.burns.2013.11.024

45. Burns-Nader S, Joe L, Pinion K. Computer tablet distraction reduces pain and anxiety in pediatric burn patients undergoing hydrotherapy: a randomized trial. Burns. 2017;43(6):1203-11. https://doi.org/10.1016/j.burns. 2017.02.015.

46. Chester S, Tyack Z, De Young A, Kipping B, Griffin B, Stockton K, et al. Efficacy of hypnosis on pain, wound-healing, anxiety, and stress in children with acute burn injuries: a randomized controlled trial. Pain. 2018;159:1790801. https://doi.org/10.1097/j.pain.0000000000001276.

47. Elliott C, Olson R. The management of children's distress in response to painful medical treatment for burn injuries. Behav Res Ther. 1983;21(6):675-83.

48. Foertsch C, O'Hara M, Stoddard F, Kealey G. Treatment-resistant pain and distress during pediatric burn-dressing changes. J Burn Care Rehabil. 1998; 19(3):219-24.

49. Hyland E, D'Cruz R, Harvey JG, Moir J, Parkinson C, Holland A. An assessment of early child life therapy pain and anxiety management: a prospective randomised controlled trial. Burns. 2015;41(8):1642-52. https:// doi.org/10.1016/j.burns.2015.05.017.

50. Jeffs D, Dorman D, Brown S, Files A, Graves T, Kirk E, et al. Effect of virtual reality on adolescent pain during burn wound care. J Burn Care Res. 2014. 35(5):395-408. https://doi.org/10.1097/bcr.0000000000000019.

51. Kavanagh C. Psychological intervention with the severely burned child: report of an experimental comparison of two approaches and their effects on psychological sequelae. J Am Acad Child Psychiatry. 1983;22(2):145-56.

52. Kipping B, Rodger S, Miller K, Kimble R. Virtual reality for acute pain reduction in adolescents undergoing burn wound care: a prospective randomized controlled trial. Burns. 2012;38(5):650-7. https://doi.org/10.1016/ j.burns.2011.11.010.

53. Miller K, Rodger S, Bucolo S, Greer R, Kimble R. Multi-modal distraction. Using technology to combat pain in young children with burn injuries. Burns. 2010;36(5):647-58. https://doi.org/10.1016/j.burns.2009.06.199.

54. Miller K, Rodger S, Kipping B, Kimble R. A novel technology approach to pain management in children with burns: a prospective randomized controlled trial. Burns. 2011;37(3):395-405. https://doi.org/10.1016/j.burns.2010.12.008.

55. Moore E, Bennett K, Dietrich M, Wells N. The effect of directed medical play on young children's pain and distress during burn wound care. J Pediatr Health Care. 2015;29(3):265-73. https://doi.org/10.1016/j.pedhc.2014.12.006.

56. Quay N, Alexander L. Preparation of burned children and their families for discharge. J Burn Care Rehabil. 1983;4(4):288-90.

57. Sveen J, Andersson G, Buhrman B, Sjöberg F, Willebrand M. Internet-based information and support program for parents of children with burns: a randomized controlled trial. Burns. 2017;43:583-91. https://doi.org/10.1016/j. burns.2016.08.039

58. van der Heijden M, Jeekel J, Rode H, Cox S, van Rosmalen J, Hunink M, et al. Can live music therapy reduce distress and pain in children with burns after wound care procedures? A randomized controlled trial. Burns. 2018:44(4): 823-33. https://doi.org/10.1016/j.burns.2017.12.013.

59. Whitehead-Pleaux A, Baryza M, Sheridan R. The effects of music therapy on pediatric patients' pain and anxiety during donor site dressing change. J Music Ther. 2006;43(2):136-53.

60. Hanson M, Gauld M, Wathen C, MacMillan H. Nonpharmacological interventions for acute wound care distress in pediatric patients with burn injury: a systematic review. J Burn Care Res. 2008;29(5):730-41. https://doi. org/10.1097/BCR.0b013e318184812e.

61. Morris L, Louw Q, Grimmer-Somers K. The effectiveness of virtual reality on reducing pain and anxiety in burn injury patients: a systematic review. Clin J Pain. 2009;25(9):815-26. https://doi.org/10.1097/AJP.0b013e3181aaa909.

62. Jenkinson E, Williamson H, Byron-Daniel J, Moss T. Systematic review: psychosocial interventions for children and young people with visible differences resulting from appearance altering conditions, injury, or treatment effects. J Pediatr Psychol. 2015;40(10):1017-33. https://doi.org/10. 1093/jpepsy/jsv048.

63. Rosenbloom B, Khan S, McCartney C, Katz J. Systematic review of persistent pain and psychological outcomes following traumatic musculoskeletal injury. J Pain Res. 2013;6:39-51. https://doi.org/10.2147/JPR.S38878.

64. Hornsby N, Blom L, Sengoelge M. Psychosocial interventions targeting recovery in child and adolescent Burns: a systematic review. J Pediatr Psychol. 2019;45:1-19. https://doi.org/10.1093/jpepsy/jsz087.
65. De Young A, Hendrikz J, Kenardy J, Cobham V, Kimble R. Prospective evaluation of parent distress following pediatric burns and identification of risk factors for young child and parent posttraumatic stress disorder. J Child Adolesc Psychopharmacol. 2014;24(1):9-17. https://doi.org/10.1089/cap.2013. 0066

66. Bonham A. Procedural pain in children with burns. Part 2: nursing management of children in pain. Int J Trauma Nurs. 1996;2(3):74-7.

67. Cahners S. Coping as the parent of a burned child. In: Bernstein N, Breslau a, Graham J, editors. Coping strategies for burn survivors and their families. 123-125. New York: Praeger; 1988.

68. Levinson P, Ousterhout D. Art and play therapy with pediatric burn patients. J Burn Care Rehabil. 1980;1(1):42-7. https://doi.org/10.1097/00004630198009000-00008

69. Rivlin E, Forshaw A, Polowyj G, Woodruff B. A multidisciplinary group approach to counselling the parents of burned children. Burns. 1986;12(7): 479-83. https://doi.org/10.1016/0305-4179(86)90073-2.

70. Russell J. Art Ther on a hospital burn unit: a step towards healing and recovery. J Am Art Ther Assoc. 1995;12(1):39. https://doi.org/10.1080/ 07421656.1995.10759122.

\section{Publisher's Note}

Springer Nature remains neutral with regard to jurisdictional claims in published maps and institutional affiliations.
Ready to submit your research? Choose BMC and benefit from:

- fast, convenient online submission

- thorough peer review by experienced researchers in your field

- rapid publication on acceptance

- support for research data, including large and complex data types

- gold Open Access which fosters wider collaboration and increased citations

- maximum visibility for your research: over $100 \mathrm{M}$ website views per year

At $\mathrm{BMC}$, research is always in progress.

Learn more biomedcentral.com/submissions 